Supporting Information for

\title{
Rhodium-Catalyzed Carbonylative Synthesis of Aryl Salicylates from Unactivated Phenols
}

\author{
Han-Jun Ai, Youcan Zhang, Fengqian Zhao and Xiao-Feng $\mathrm{Wu}^{*}$ \\ Leibniz-Institut für Katalyse e.V. an der UniversitätRostock, Albert-Einstein-Straße 29a, 18059 Rostock, \\ Germany,E-Mail:xiao-feng.wu@catalysis.de \\ Table of Contents
}

General Remarks.......................................................................................................................................

General Procedure for Synthesis of Phenyl Salicylate............................................................................S3

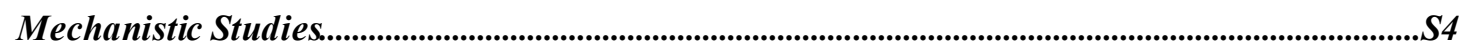

Procedure and Characterization of the Products..................................................................................... 7

Analytic Date and NMR Spectra of the Products........................................................................... S13 


\section{General Remarks}

Reagents and solvents: Unless otherwise noted, the chemicals were commercially available from Sigma-Aldrich, TCI or Alfa Aesar and were used without further purification. Dioxane was bought from Alfa Aesar, HPLC grade, 99\% min, packaged under argon in resealable ChemSeal bottles. The reaction does not require the glovebox.

Purification: The products were isolated from the reaction mixture by column chromatography on silica gel 60, 0.063-0.2 mm, 70-230 mesh (Merck). Gradient flash chromatography was conducted eluting with PE/EA, PE refers to pentane and EA refers to ethyl acetate, they were listed as volume/volume ratios.

Data collection: GC-yields were calculated using hexadecane as internal standard. GC analysis was performed on an Agilent HP-7890A instrument with FID detector and HP-5 capillary column (polydimethylsiloxane with $5 \%$ phenyl groups, $30 \mathrm{~m}, 0.32 \mathrm{~mm}$ i.d., $0.25 \mu \mathrm{m}$ film thickness) using argon as carrier gas. High resolution mass spectra (HRMS) were recorded on Agilent 6210. NMR spectra were recorded on Bruker A vance 300 and Bruker ARX 400 spectrometers. Chemical shifts (ppm) are given relative to solvent: references for $\mathrm{CDCl}_{3}$ were $7.26 \mathrm{ppm}\left({ }^{1} \mathrm{H} \mathrm{NMR}\right)$ and $77.00 \mathrm{ppm}\left({ }^{13} \mathrm{C} \mathrm{NMR}\right)$. All measurements were carried out at room temperature unless otherwise stated. 


\section{General Procedure for Synthesis of Phenyl Salicylate}

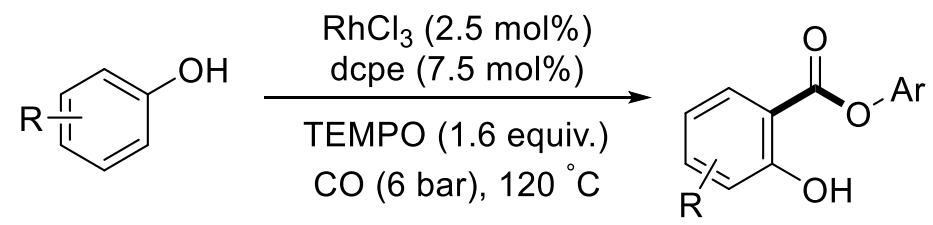

Room temperature under air, an oven-dried 4-mL vial was charged with $\mathrm{RhCl}_{3}(0.0125 \mathrm{mmol}$, $2.5 \mathrm{~mol} \%)$, dcpe (0.0375 mmol, $7.5 \mathrm{~mol} \%)$, phenol (0.5 mmol, 1 equiv), $3 \AA$ MS (10 mg. $3 \AA$ MS was heated for about 5 min with a heat gun under vacuum before it's used.), TEMPO (0.8 mmol) (Please follow this order, the order of addition will affect the reaction result) and a 1.0 $\mathrm{cm}$ stirring bar. Then dioxane $(0.4 \mathrm{~mL})$ and toluene $(0.6 \mathrm{~mL})$ was added by syringe. The vial was then capped (with a needle) and placed in an aluminum rack, which was transferred into a $300 \mathrm{~mL}$ autoclave of the 4560 series from the Parr Instruments ${ }^{\circledR}$. After flushing the autoclave three times with $\mathrm{CO}$, a pressure of 6 bar of $\mathrm{CO}$ was adjusted at ambient temperature. (NOTE: Carbon monoxide should only be handled in a well-ventilated fume hood). Then, the reaction was performed for $24 \mathrm{~h}$ at $120^{\circ} \mathrm{C}$ (aluminum block). After the reaction completed, the autoclave was cooled down to room temperature and the pressure was released carefully. The crude product was purified by silica gel chromatography (PE/EA) to afford the corresponding products. (NOTE: some products are volatile. So appropriate amount of DCM could be added during rotary evaporation and the product should not be vacuum dried for a long time).

5 mmol scale: Room temperature under air, an oven-dried 12-mL vial was charged with $\mathrm{RhCl}_{3}$ (2.5 mol\%), dcpe (7.5 mol\%), phenol (5 mmol, 1 equiv), $3 \AA$ MS (20 mg. $3 \AA$ MS was heated for about $5 \mathrm{~min}$ with a heat gun under vacuum before it's used.), TEMPO (8 mmol) and a 1.0 $\mathrm{cm}$ stirring bar. Then dioxane $(4 \mathrm{~mL})$ and toluene $(6 \mathrm{~mL})$ was added by syringe. The vial was then capped (with a needle) and placed in an aluminum rack, which was transferred into a 300 $\mathrm{mL}$ autoclave of the 4560 series from the Parr Instruments ${ }^{\circledR}$. After flushing the autoclave three times with $\mathrm{CO}$, a pressure of 6 bar of $\mathrm{CO}$ was adjusted at ambient temperature. Then, the reaction was performed for $24 \mathrm{~h}$ at $120^{\circ} \mathrm{C}$ (aluminum block). After the reaction completed, the autoclave was cooled down to room temperature and the pressure was released carefully. The crude product was purified by silica gel chromatography (PE/EA) to afford the pure product in $81 \%$ yield ( $433.4 \mathrm{mg})$. 


\section{Mechanistic Studies}

\section{Control experiments}<smiles>O=C(Oc1ccccc1)Oc1ccccc1</smiles>

3

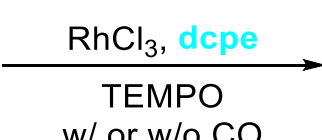

w/ or w/o CO<smiles>O=C(Oc1ccccc1)c1ccccc1O</smiles>

2, n.d.<smiles>O=C(Oc1ccccc1)Oc1ccccc1</smiles>

3, recovered $>95 \%$

Room temperature under air, an oven-dried 4-mL vial was charged with $\mathrm{RhCl}_{3}(0.0125$ mmol, 5 mol\%), dcpe (0.0375 mmol, 15 mol\%), DPC (0.25 mmol, 1 equiv), 3 Å MS (10 mg. 3 $\AA$ MS was heated for about $5 \mathrm{~min}$ with a heat gun under vacuum before it's used.), TEMPO $(0.8 \mathrm{mmol})$ (Please follow this order, the order of addition will affect the reaction result) and a $1.0 \mathrm{~cm}$ stirring bar. Then dioxane $(0.4 \mathrm{~mL})$ and toluene $(0.6 \mathrm{~mL})$ was added by syringe. The vial was then capped (with a needle) and placed in an aluminum rack, which was transferred into a $300 \mathrm{~mL}$ autoclave of the 4560 series from the Parr Instruments ${ }^{\circledR}$. After flushing the autoclave three times with $\mathrm{CO}$, a pressure of 6 bar of $\mathrm{CO}$ was adjusted at ambient temperature. (NOTE: Carbon monoxide should only be handled in a well-ventilated fume hood). Then, the reaction was performed for $24 \mathrm{~h}$ at $120^{\circ} \mathrm{C}$ (aluminum block). After the reaction completed, let it cool to room temperature, the $\mathrm{CO}$ gas was released. Then $10 \mu \mathrm{L}$ of hexadecane was added as internal standard and a proper amount of solution was taken after it was well mixed for GC and GC-MS analysis. The result is shown above. AND the similar result was obtained when the reaction performed under $\mathrm{N}_{2}$.<smiles>O=C(Oc1ccccc1)c1ccccc1O</smiles>

2

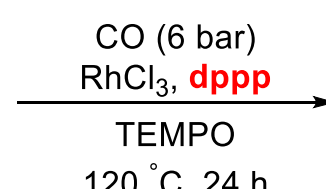

$120^{\circ} \mathrm{C}, 24 \mathrm{~h}$<smiles>O=C(Oc1ccccc1)Oc1ccccc1</smiles>

3, n.d.<smiles>O=C(Oc1ccccc1)c1ccccc1O</smiles>

2, recovered $50 \%$

Room temperature under air, an oven-dried 4-mL vial was charged with $\mathrm{RhCl}_{3}(0.0125$ mmol, 5 mol\%), dppp (0.0375 mmol, 15 mol\%), 2 (0.25 mmol, 1 equiv), 3 A MS (10 mg. $3 \AA$ MS was heated for about 5 min with a heat gun under vacuum before it's used.), TEMPO (0.8 mmol) (Please follow this order, the order of addition will affect the reaction result) and a 1.0 $\mathrm{cm}$ stirring bar. Then dioxane $(0.4 \mathrm{~mL})$ and toluene $(0.6 \mathrm{~mL})$ was added by syringe. The vial was then capped (with a needle) and placed in an aluminum rack, which was transferred into a 
$300 \mathrm{~mL}$ autoclave of the 4560 series from the Parr Instruments ${ }^{\circledR}$. After flushing the autoclave three times with $\mathrm{CO}$, a pressure of 6 bar of $\mathrm{CO}$ was adjusted at ambient temperature. (NOTE: Carbon monoxide should only be handled in a well-ventilated fume hood). Then, the reaction was performed for $24 \mathrm{~h}$ at $120^{\circ} \mathrm{C}$. After the reaction completed, let it cool to room temperature, the $\mathrm{CO}$ gas was released. Then $10 \mu \mathrm{L}$ of hexadecane was added as internal standard and a proper amount of solution was taken after it was well mixed for GC and GC-MS analysis. The result is shown above.

\section{Deuterated experiments}<smiles>[13CH3]c1ccccc1</smiles>

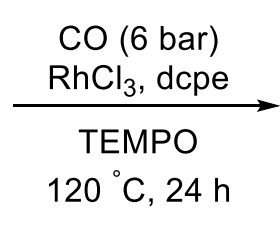<smiles>O=C(Oc1ccccc1)c1ccccc1O</smiles><smiles></smiles>

Room temperature under air, an oven-dried 4-mL vial was charged with $\mathrm{RhCl}_{3}(0.0125$ mmol, $2.5 \mathrm{~mol} \%$ ), dcpe (0.0375 mmol, $7.5 \mathrm{~mol} \%)$, 1-d1 (>95\% d; $0.5 \mathrm{mmol}, 1$ equiv), 3 Å MS (10 mg. $3 \AA$ MS was heated for about 5 min with a heat gun under vacuum before it's used.), TEMPO $(0.8 \mathrm{mmol})$ (Please follow this order, the order of addition will affect the reaction result) and a $1.0 \mathrm{~cm}$ stirring bar. Then dioxane $(0.4 \mathrm{~mL})$ and toluene $(0.6 \mathrm{~mL})$ was added by syringe. The vial was then capped (with a needle) and placed in an aluminumrack, which was transferred into a $300 \mathrm{~mL}$ autoclave of the 4560 series from the Parr Instruments ${ }^{\circledR}$. After flushing the autoclave three times with $\mathrm{CO}$, a pressure of 6 bar of $\mathrm{CO}$ was adjusted at ambient temperature. (NOTE: Carbon monoxide should only be handled in a well-ventilated fume hood). Then, the reaction was performed for $24 \mathrm{~h}$ at $120^{\circ} \mathrm{C}$ (aluminum block). After the reaction completed, let it cool to room temperature, the $\mathrm{CO}$ gas was released. Then $10 \mu \mathrm{L}$ of hexadecane was added as internal standard and a proper amount of solution was taken after it was well mixed for GC and GC-MS analysis. Subsequently, the crude product was purified by silica gel chromatography (PE/EA) to afford the corresponding products which next analyzed by NMR spectrometers. The result was shown above. 

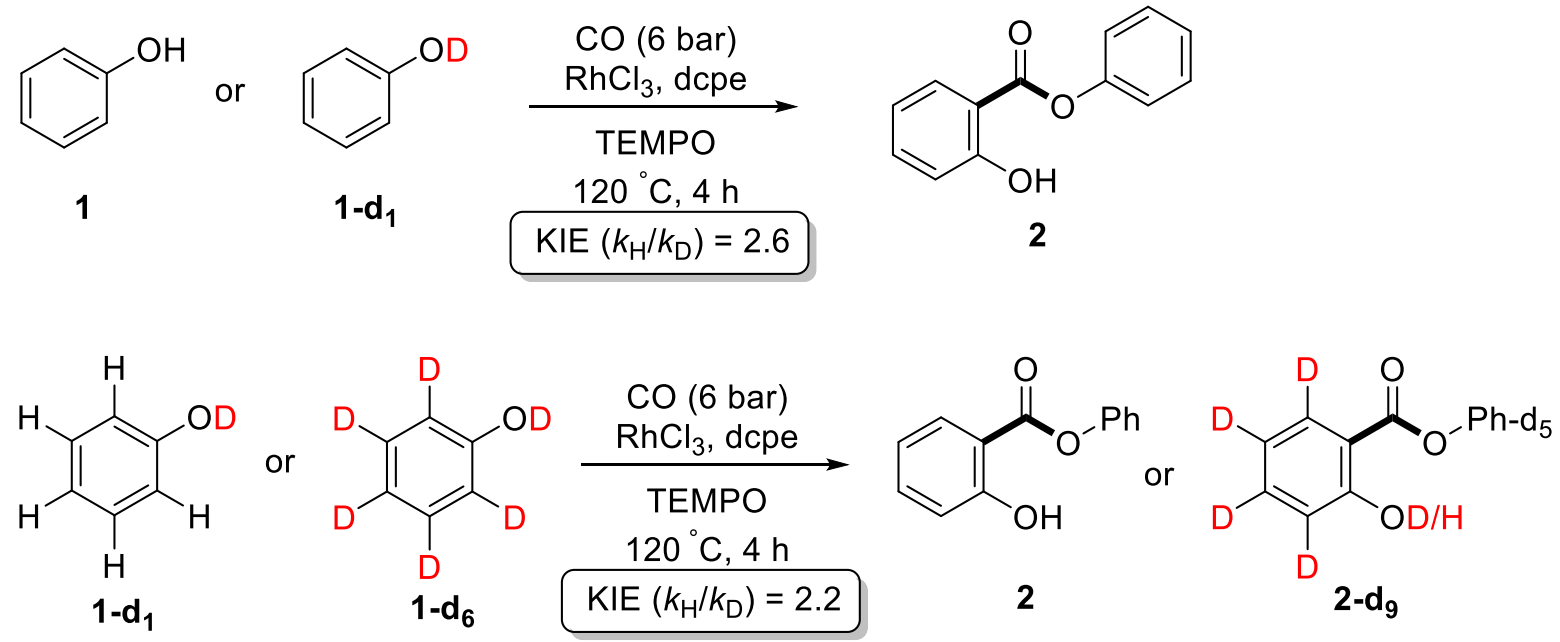

Room temperature under air, three oven-dried 4-mL vials were charged with $\mathrm{RhCl}_{3}$ (0.0125 mmol, $2.5 \mathrm{~mol} \%)$, depe $(0.0375 \mathrm{mmol}, 7.5 \mathrm{~mol} \%)$, and $\mathbf{1}, \mathbf{1 - d _ { 1 }}, \mathbf{1}-\mathbf{d}_{\mathbf{6}}(0.5 \mathrm{mmol}, 1$ equiv) respectively. Then $3 \AA$ MS (10 mg. $3 \AA$ MS was heated for about 5 min with a heat gun under vacuum before it's used.), TEMPO $(0.8 \mathrm{mmol})$ (Please follow this order, the order of addition will affect the reaction result), a $1.0 \mathrm{~cm}$ stirring bar, dioxane $(0.4 \mathrm{~mL})$ and toluene $(0.6 \mathrm{~mL})$ were added. The vials were then capped (with a needle) and placed in an aluminum rack, which was transferred into a $300 \mathrm{~mL}$ autoclave of the 4560 series from the Parr Instruments ${ }^{\circledR}$. After flushing the autoclave three times with $\mathrm{CO}$, a pressure of 6 bar of $\mathrm{CO}$ was adjusted at ambient temperature. (NOTE: Carbon monoxide should only be handled in a well-ventilated fume hood). Then, the reaction was performed for $4 \mathrm{~h}$ at $120{ }^{\circ} \mathrm{C}$ (aluminum block). After the reaction completed, let it cool to room temperature, the $\mathrm{CO}$ gas was released. Then $10 \mu \mathrm{L}$ of hexadecane was added as internal standard and a proper amount of solutions were taken after it was well mixed for GC and GC-MS analysis. The yield of the obtained products was 34\%, $13 \%$ and $6 \%$ respectively. 
<smiles>O=C(Oc1ccccc1)c1ccccc1O</smiles>

Phenyl 2-hydroxybenzoate (2). Prepared according to general procedure using phenol (47.1 $\mathrm{mg}, 0.5 \mathrm{mmol})$. The crude product was purified by silica gel chromatography $(\mathrm{PE} / \mathrm{EA}=50: 1)$ to afford the title compound as a colorless oil $(42.8 \mathrm{mg}, 80 \%$ yield).

${ }^{1} \mathbf{H}$ NMR $\left(300 \mathrm{MHz}, \mathrm{CDCl}_{3}\right) \delta 10.43(\mathrm{~s}, 1 \mathrm{H}), 7.99(\mathrm{~d}, \mathrm{~J}=8.0 \mathrm{~Hz}, 1 \mathrm{H}), 7.44(\mathrm{t}, \mathrm{J}=8.5 \mathrm{~Hz}, 1 \mathrm{H})$, $7.40-7.31(\mathrm{~m}, 2 \mathrm{H}), 7.26-7.17(\mathrm{~m}, 1 \mathrm{H}), 7.15-7.08(\mathrm{~m}, 2 \mathrm{H}), 6.95(\mathrm{~d}, \mathrm{~J}=8.5 \mathrm{~Hz}, 1 \mathrm{H}), 6.92(\mathrm{t}$, $\mathrm{J}=6 \mathrm{~Hz}, 1 \mathrm{H})$.

${ }^{13}$ C NMR $\left(75 \mathrm{MHz}, \mathrm{CDCl}_{3}\right) \delta 168.9,162.2,150.1,136.4,130.3,129.6,126.3,121.6,119.4$, $117.8,111.8$.

HRMS (EI) m/z: calcd. for $\left[\mathrm{C}_{13} \mathrm{H}_{10} \mathrm{O}_{3}\right]^{+} 214.0625$; found 214.0624 .<smiles>COc1ccc(OC(=O)c2cc(OC)ccc2O)cc1</smiles>

4-Methoxyphenyl 2-hydroxy-5-methoxybenzoate (4). Prepared according to general proced ure using 4-methoxyphenol $(62.1 \mathrm{mg}, 0.5 \mathrm{mmol})$. The crude product was purified by silica gel chromatography $(\mathrm{PE} / \mathrm{EA}=5: 1)$ to afford the title compound as a colorless oil $(53.3 \mathrm{mg}, 78 \%$ yield).

${ }^{1} \mathbf{H}$ NMR $\left(400 \mathrm{MHz}, \mathrm{CDCl}_{3}\right) \delta 10.18(\mathrm{~s}, 1 \mathrm{H}), 7.51(\mathrm{~d}, J=3.0 \mathrm{~Hz}, 1 \mathrm{H}), 7.21-7.13(\mathrm{~m}, 2 \mathrm{H}), 7.13$ $(\mathrm{s}, 1 \mathrm{H}), 6.98(\mathrm{~m}, 3 \mathrm{H}), 3.85(\mathrm{~s}, 3 \mathrm{H}), 3.84(\mathrm{~s}, 3 \mathrm{H})$.

${ }^{13} \mathrm{C}$ NMR $\left(101 \mathrm{MHz}, \mathrm{CDCl}_{3}\right) \delta 169.0,157.6,156.7,152.1,143.4,124.9,122.4,118.8,114.6$, $111.9,111.2,55.9,55.6$.

HRMS (EI) m/z: calcd. for $\left[\mathrm{C}_{15} \mathrm{H}_{14} \mathrm{O}_{5}\right]^{+} 274.0836$; found 274.0835 .<smiles>CC(C)(C)c1ccc(OC(=O)c2cc(C(C)(C)C)ccc2O)cc1</smiles>

4-(tert-Butyl)phenyl 5-(tert-butyl)-2-hydroxybenzoate (5). Prepared according to general pr ocedure using 4-(tert-butyl)phenol $(75.1 \mathrm{mg}, 0.5 \mathrm{mmol})$. The crude product was purified by sil ica gel chromatography $(\mathrm{PE} / \mathrm{EA}=50: 1)$ to afford the title compound as a white solid $(63 \mathrm{mg}$, $77 \%$ yield).

${ }^{1} \mathbf{H}$ NMR $\left(300 \mathrm{MHz}, \mathrm{CDCl}_{3}\right) \delta 10.32(\mathrm{~s}, 1 \mathrm{H}), 7.95(\mathrm{~d}, J=2.5 \mathrm{~Hz}, 1 \mathrm{H}), 7.50(\mathrm{dd}, J=8.5,2.5$ $\mathrm{Hz}, 1 \mathrm{H}), 7.37(\mathrm{~d}, J=8.5 \mathrm{~Hz}, 2 \mathrm{H}), 7.05(\mathrm{~d}, J=8.5 \mathrm{~Hz}, 2 \mathrm{H}), 6.89(\mathrm{~d}, J=8.5 \mathrm{~Hz}, 1 \mathrm{H}), 1.26$ (s, 9H), 1.25 (s, 9H).

${ }^{13} \mathbf{C}$ NMR $\left(75 \mathrm{MHz}, \mathrm{CDCl}_{3}\right) \delta 169.3,160.1,149.2,147.8,142.2,134.1,126.5,126.2,121.0$, 117.4, 111.0, 34.5, 34.1,31.4, 31.3.

HRMS (ESI-TOF) m/z: calcd. for $\left[\mathrm{C}_{21} \mathrm{H}_{26} \mathrm{O}_{3}+\mathrm{H}\right]+327.1960$; found 327.1960. 
<smiles>CC(C)(c1ccccc1)c1ccc(OC(=O)c2cc(C(C)(C)c3ccccc3)ccc2O)cc1</smiles>

4-(2-Phenylpropan-2-yl)phenyl 2-hydroxy-5-(2-phenylpropan-2-yl)benzoate (6). Prepared according to general procedure using 4-(2-phenylpropan-2-yl)phenol (106.2 mg, $0.5 \mathrm{mmol}$ ). The crude product was purified by silica gel chromatography $(\mathrm{PE} / \mathrm{EA}=50: 1)$ to afford the titl e compound as a white solid ( $82 \mathrm{mg}, 73 \%$ yield).

${ }^{1} \mathbf{H}$ NMR $\left(400 \mathrm{MHz}, \mathrm{CDCl}_{3}\right) \delta 10.36(\mathrm{~s}, 1 \mathrm{H}), 7.93(\mathrm{~d}, J=2.5 \mathrm{~Hz}, 1 \mathrm{H}), 7.25-7.13(\mathrm{~m}, 11 \mathrm{H})$, $7.12-7.04(\mathrm{~m}, 2 \mathrm{H}), 7.01(\mathrm{~d}, J=8.5 \mathrm{~Hz}, 2 \mathrm{H}), 6.83(\mathrm{~d}, J=8.5 \mathrm{~Hz}, 1 \mathrm{H}), 1.61(\mathrm{~s}, 6 \mathrm{H}), 1.61(\mathrm{~s}$, $6 \mathrm{H})$.

${ }^{13}$ C NMR $\left(101 \mathrm{MHz}, \mathrm{CDCl}_{3}\right) \delta 169.2,160.3,150.2,150.0,148.9,147.9,141.8,136.2,128.1$, 128.1, 128.0, 126.9, 126.7, 126.6, 125.8, 125.8, 121.0, 117.6, 110.8, 42.8, 42.3, 30.8, 30.7.

HRMS (ESI-TOF) m/z: calcd. for $\left[\mathrm{C}_{31} \mathrm{H}_{30} \mathrm{O}_{3}+\mathrm{H}\right]+451.2273$; found 451.2274 .<smiles>O=C(Oc1ccc(-c2ccccc2)cc1)c1cc(-c2ccccc2)ccc1O</smiles>

[1,1'-Biphenyl]-4-yl 4-hydroxy-[1,1'-biphenyl]-3-carboxylate (7). Prepared according to ge neral procedure using [1,1'-biphenyl]-4-ol $(85 \mathrm{mg}, 0.5 \mathrm{mmol})$. The crude product was purified by silica gel chromatography (PE/EA $=50: 1)$ to afford the title compound as a white solid (64 $\mathrm{mg}, 70 \%$ yield).

${ }^{1} \mathbf{H}$ NMR $\left(400 \mathrm{MHz}, \mathrm{CDCl}_{3}\right) \delta 10.43(\mathrm{~s}, 1 \mathrm{H}), 8.19(\mathrm{~d}, J=2.5 \mathrm{~Hz}, 1 \mathrm{H}), 7.63(\mathrm{dd}, J=8.5,2.5$ $\mathrm{Hz}, 1 \mathrm{H}), 7.51(\mathrm{~d}, J=8.5 \mathrm{~Hz}, 2 \mathrm{H}), 7.51-7.37(\mathrm{~m}, 4 \mathrm{H}), 7.36-7.24(\mathrm{~m}, 4 \mathrm{H}), 7.26-7.18(\mathrm{~m}, 2 \mathrm{H})$, $7.15(\mathrm{~d}, J=8.5 \mathrm{~Hz}, 2 \mathrm{H}), 6.99(\mathrm{~d}, J=8.5 \mathrm{~Hz}, 1 \mathrm{H})$.

${ }^{13} \mathrm{C}$ NMR $\left(75 \mathrm{MHz}, \mathrm{CDCl}_{3}\right) \delta 168.9,161.5,149.3,140.0,139.6,139.5,135.2,132.7,128.8$, $128.7,128.4,128.2,127.4,127.1,127.0,126.6,121.8,121.0,118.2,111.8$.

HRMS (EI) m/z: calcd. for $\left[\mathrm{C}_{25} \mathrm{H}_{18} \mathrm{O}_{3}\right]+366.1251$; found 366.1248 .<smiles>CCc1ccc(OC(=O)c2cc(CC)ccc2O)cc1</smiles>

4-Ethylphenyl 5-ethyl-2-hydroxybenzoate (8). Prepared according to general procedure usin g 4-ethylphenol $(61.1 \mathrm{mg}, 0.5 \mathrm{mmol})$. The crude product was purified by silica gel chromatogr aphy $(\mathrm{PE} / \mathrm{EA}=50: 1)$ to afford the title compound as a colorless oil ( $41.7 \mathrm{mg}, 62 \%$ yield $)$.

${ }^{1} \mathbf{H}$ NMR $\left(300 \mathrm{MHz}, \mathrm{CDCl}_{3}\right) \delta 10.43(\mathrm{~s}, 1 \mathrm{H}), 7.93(\mathrm{~d}, J=2.0 \mathrm{~Hz}, 1 \mathrm{H}), 7.43(\mathrm{dd}, J=8.5,2.0$ $\mathrm{Hz}, 1 \mathrm{H}), 7.32(\mathrm{~d}, J=8.5 \mathrm{~Hz}, 2 \mathrm{H}), 7.22-7.11(\mathrm{~m}, 2 \mathrm{H}), 7.02(\mathrm{~d}, J=8.5 \mathrm{~Hz}, 1 \mathrm{H}), 2.81-2.62(\mathrm{~m}$, $4 \mathrm{H}), 1.35-1.27(\mathrm{~m}, 6 \mathrm{H})$.

${ }^{13} \mathbf{C}$ NMR $\left(75 \mathrm{MHz}, \mathrm{CDCl}_{3}\right) \delta 169.2,160.3,148.0,142.3,136.3,135.1,128.9,128.8,121.4$, 120.6, 117.6, 111.5, 28.3, 27.9, 15.7, 15.5.

HRMS (EI) m/z: calcd. for $\left[\mathrm{C}_{17} \mathrm{H}_{18} \mathrm{O}_{3}\right]+270.1251$; found 270.1248 . 
<smiles>Cc1ccc(OC(=O)c2cc(C)ccc2O)cc1</smiles>

$p$-Tolyl 2-hy droxy-5-methylbenzoate (9). Prepared according to general procedure using $p$-c resol (54.1 mg, $0.5 \mathrm{mmol})$. The crude product was purified by silica gel chromatography (PE/ $\mathrm{EA}=50: 1)$ to afford the title compound as a colorless oil $(36.3 \mathrm{mg}, 60 \%$ yield $)$.

${ }^{1} \mathbf{H}$ NMR $\left(300 \mathrm{MHz}, \mathrm{CDCl}_{3}\right) \delta 10.27(\mathrm{~s}, 1 \mathrm{H}), 7.79(\mathrm{~d}, J=2.5 \mathrm{~Hz}, 1 \mathrm{H}), 7.27(\mathrm{dd}, J=8.5,2.5$ $\mathrm{Hz}, 1 \mathrm{H}), 7.17(\mathrm{~d}, J=8.5 \mathrm{~Hz}, 2 \mathrm{H}), 7.01(\mathrm{~d}, J=8.5 \mathrm{~Hz}, 2 \mathrm{H}), 6.86(\mathrm{~d}, J=8.5 \mathrm{~Hz}, 1 \mathrm{H}), 2.31(\mathrm{~s}$, $3 \mathrm{H}), 2.26(\mathrm{~s}, 3 \mathrm{H})$.

${ }^{13} \mathrm{C}$ NMR $\left(75 \mathrm{MHz}, \mathrm{CDCl}_{3}\right) \delta 169.2,160.1,147.9,137.4,136.0,130.1,129.9,128.6,121.3$, 117.6, 111.4, 20.9, 20.4.

HRMS (EI) m/z: calcd. for $\left[\mathrm{C}_{15} \mathrm{H}_{14} \mathrm{O}_{3}\right]+242.0938$; found 242.0940 .<smiles>CC(=O)CCc1ccc(OC(=O)c2cc(CCC(C)=O)ccc2O)cc1</smiles>

4-(3-Oxobutyl)phenyl 2-hydroxy-5-(3-oxobutyl)benzoate (10). Prepared according to gener al procedure using raspberry ketone $(82.1 \mathrm{mg}, 0.5 \mathrm{mmol})$. The crude product was purified by $\mathrm{s}$ ilica gel chromatography $(\mathrm{PE} / \mathrm{EA}=20: 1)$ to afford the title compound as a white solid $(74.6 \mathrm{~m}$ g, $84 \%$ yield).

${ }^{1} \mathbf{H}$ NMR $\left(400 \mathrm{MHz}, \mathrm{CDCl}_{3}\right) \delta 10.38(\mathrm{~s}, 1 \mathrm{H}), 7.88(\mathrm{~d}, J=2.5 \mathrm{~Hz}, 1 \mathrm{H}), 7.39(\mathrm{dd}, J=8.5,2.5$ $\mathrm{Hz}, 1 \mathrm{H}), 7.32-7.22(\mathrm{~m}, 2 \mathrm{H}), 7.13(\mathrm{~d}, J=8.5 \mathrm{~Hz}, 2 \mathrm{H}), 6.97(\mathrm{~d}, J=8.5 \mathrm{~Hz}, 1 \mathrm{H}), 2.92(\mathrm{~m}, 4 \mathrm{H})$, $2.85-2.75(\mathrm{~m}, 4 \mathrm{H}), 2.18(\mathrm{~s}, 6 \mathrm{H})$.

${ }^{13} \mathrm{C}$ NMR $\left(101 \mathrm{MHz}, \mathrm{CDCl}_{3}\right) \delta 207.5,207.5,168.9,160.5,148.3,139.2,136.7,132.0,129.4$, $129.4,121.5,117.9,111.5,45.0,45.0,30.1,30.0,29.0,28.6$.

HRMS (ESI-TOF) m/z: calcd. for $\left[\mathrm{C}_{21} \mathrm{H}_{22} \mathrm{O}_{5}+\mathrm{Na}\right]^{+} 377.1364$; found 377.1365 .<smiles>O=C(Oc1ccc(F)cc1)c1cc(F)ccc1O</smiles>

4-Fluorophenyl 5-fluoro-2-hydroxybenzoate (11). Prepared according to general procedure using 4-fluorophenol $(56.1 \mathrm{mg}, 0.5 \mathrm{mmol})$ at $130^{\circ} \mathrm{C}$. The crude product was purified by silica gel chromatography $(\mathrm{PE} / \mathrm{EA}=50: 1)$ to afford the title compound as a white solid $(42.1 \mathrm{mg}, 6$ $7 \%$ yield).

${ }^{1} \mathbf{H}$ NMR $\left(300 \mathrm{MHz}, \mathrm{CDCl}_{3}\right) \delta 10.12(\mathrm{~s}, 1 \mathrm{H}), 7.63(\mathrm{dd}, J=8.5,3.0 \mathrm{~Hz}, 1 \mathrm{H}), 7.24-7.15(\mathrm{~m}$, $1 \mathrm{H}), 7.14-7.01(\mathrm{~m}, 4 \mathrm{H}), 6.92(\mathrm{dd}, J=9.0,4.5 \mathrm{~Hz}, 1 \mathrm{H})$.

${ }^{13}$ C NMR $\left(75 \mathrm{MHz}, \mathrm{CDCl}_{3}\right) \delta 168.1, \delta 160.62(\mathrm{~d}, J=245.6 \mathrm{~Hz}), 158.49(\mathrm{~d}, J=1.7 \mathrm{~Hz}), 155.21$ $(\mathrm{d}, J=239.1 \mathrm{~Hz}), 145.65(\mathrm{~d}, J=3.0 \mathrm{~Hz}), 124.26(\mathrm{~d}, J=23.7 \mathrm{~Hz}), 122.97(\mathrm{~d}, J=8.6 \mathrm{~Hz}), 119.21$ $(\mathrm{d}, J=7.4 \mathrm{~Hz}), 116.39(\mathrm{~d}, J=23.7 \mathrm{~Hz}), 115.30(\mathrm{~d}, J=24.4 \mathrm{~Hz}), 111.49(\mathrm{~d}, J=7.7 \mathrm{~Hz})$.

${ }^{19}$ F NMR $\left(282 \mathrm{MHz}, \mathrm{CDCl}_{3}\right) \delta-115.71,-123.45$.

HRMS (EI) m/z: calcd. for $\left[\mathrm{C}_{13} \mathrm{H}_{8} \mathrm{O}_{3} \mathrm{~F}_{2}\right]^{+} 250.0436$; found 250.0435 . 
<smiles>O=C(Oc1ccc(Cl)cc1)c1cc(Cl)ccc1O</smiles>

4-Chlorophenyl 5-chloro-2-hydroxybenzoate (12). Prepared according to general procedure using 4-chlorophenol ( $64.3 \mathrm{mg}, 0.5 \mathrm{mmol})$. The crude product was purified by silica gel chro matography $(\mathrm{PE} / \mathrm{EA}=50: 1)$ to afford the title compound as a light yellow solid $(42.7 \mathrm{mg}, 61 \%$ yield).

${ }^{1} \mathbf{H}$ NMR $\left(300 \mathrm{MHz}, \mathrm{CDCl}_{3}\right) \delta 10.32(\mathrm{~s}, 1 \mathrm{H}), 8.02(\mathrm{~d}, J=2.5 \mathrm{~Hz}, 1 \mathrm{H}), 7.49(\mathrm{dd}, J=9.0,2.5$ $\mathrm{Hz}, 1 \mathrm{H}), 7.46-7.39(\mathrm{~m}, 2 \mathrm{H}), 7.22-7.11(\mathrm{~m}, 2 \mathrm{H}), 7.00(\mathrm{~d}, J=9.0 \mathrm{~Hz}, 1 \mathrm{H})$.

${ }^{13} \mathbf{C}$ NMR $\left(75 \mathrm{MHz}, \mathrm{CDCl}_{3}\right) \delta 167.7,160.8,148.3,136.6,132.1,129.8,129.5,124.3,122.9$, 122.2, $119.5,112.4$.

HRMS (EI) m/z: calcd. for $\left[\mathrm{C}_{13} \mathrm{H}_{8} \mathrm{O}_{3} \mathrm{Cl}_{2}\right]+281.9845$; found 281.9848 .<smiles>Cc1cccc(OC(=O)c2ccc(C)cc2O)c1</smiles>

$13,58 \%$ yield $(4-, 6-=76 \%, 24 \%)$

m-Tolyl 2-hydroxy-4-methylbenzoate AND m-tolyl 2-hydroxy-6-methylbenzoate (13). Pre pared according to general procedure using $m$-cresol $(52.5 \mu \mathrm{L}, 0.5 \mathrm{mmol})$. The crude product was purified by silica gel chromatography $(\mathrm{PE} / \mathrm{EA}=50: 1)$ to afford the title compound as a co lorless oil (67.5 mg, $58 \%$ yield).

${ }^{1} \mathbf{H}$ NMR $\left(300 \mathrm{MHz}, \mathrm{CDCl}_{3}\right.$ ) integration of $p$-Tolyl 2-hydroxy-4-methylbenzoate AND $p$-tolyl 2-hydroxy-6-methylbenzoate: $\delta 11.05(\mathrm{~s}, 0.31 \mathrm{H}), 10.50(\mathrm{~s}, 1 \mathrm{H}), 7.95(\mathrm{~d}, J=8.0 \mathrm{~Hz}, 1 \mathrm{H}), 7.44$ - $7.29(\mathrm{~m}, 2.16 \mathrm{H}), 7.20-6.98(\mathrm{~m}, 5.13 \mathrm{H}), 6.96-6.84(\mathrm{~m}, 1.39 \mathrm{H}), 6.86-6.72(\mathrm{~m}, 1.41 \mathrm{H}), 2.72$ (s, 1H), $2.42(\mathrm{~m}, 4.49 \mathrm{H}), 2.40(\mathrm{~s}, 3 \mathrm{H})$.

${ }^{13} \mathbf{C}$ NMR $\left(75 \mathrm{MHz}, \mathrm{CDCl}_{3}\right.$ ) peak picking of $p$-Tolyl 2-hydroxy-4-methylbenzoate only: $\delta$ 169.0, 162.1, 147.9, 139.8, 130.1, 129.3, 127.0, 122.2 , 120.7, 118.6, 117.9, 115.9, 109.3, 21.9, 21.3.

HRMS (EI) m/z: calcd. for $\left[\mathrm{C}_{15} \mathrm{H}_{14} \mathrm{O}_{3}\right]+242.0938$; found 242.0941 .<smiles>O=C(Oc1cccc(-c2ccccc2)c1)c1ccc(-c2ccccc2)cc1O</smiles>

$14,74 \%$ yield $(4-, 6-=92 \%, 8 \%)$

[1,1'-Biphenyl]-3-yl 3-hydroxy-[1,1'-biphenyl]-4-carboxylate AND [1,1'-biphenyl]-3-yl 3hydroxy-[1,1'-biphenyl]-2-carboxylate (14). Prepared according to general procedure using [1,1'-biphenyl]-3-ol ( $85.1 \mathrm{mg}, 0.5 \mathrm{mmol})$. The crude product was purified by silica gel chroma tography $(\mathrm{PE} / \mathrm{EA}=50: 1)$ to afford the title compound as a light yellow oil $(67.7 \mathrm{mg}, 74 \%$ yiel d).

${ }^{1} \mathbf{H}$ NMR $\left(400 \mathrm{MHz}, \mathrm{CDCl}_{3}\right.$ ) integration of [1,1'-Biphenyl]-4-yl 3-hydroxy-[1,1'-biphenyl]-4carboxylate AND [1,1'-biphenyl]-4-yl 3-hydroxy-[1,1'-biphenyl]-2-carboxylate: $\delta 10.49(\mathrm{~s}, 1 \mathrm{H})$, $10.44(\mathrm{~s}, 0.08 \mathrm{H}), 8.24(\mathrm{~d}, J=2.5 \mathrm{~Hz}, 0.09 \mathrm{H}), 8.05(\mathrm{~d}, J=8.5 \mathrm{~Hz}, 1 \mathrm{H}), 7.69$ (dd, $J=8.5,2.5 \mathrm{~Hz}$, $0.09 \mathrm{H}), 7.58-7.48(\mathrm{~m}, 5.8 \mathrm{H}), 7.40-7.31(\mathrm{~m}, 7.6 \mathrm{H}), 7.31-7.23(\mathrm{~m}, 1.4 \mathrm{H}), 7.19(\mathrm{~d}, J=1.5 \mathrm{~Hz}$, $1.2 \mathrm{H}), 7.16-7.08(\mathrm{~m}, 2.3 \mathrm{H}), 7.04(\mathrm{~d}, J=8.5 \mathrm{~Hz}, 0.15 \mathrm{H})$. 
${ }^{13} \mathbf{C}$ NMR $\left(101 \mathrm{MHz}, \mathrm{CDCl}_{3}\right)$ peak picking of [1,1'-Biphenyl]-4-yl 3-hydroxy-[1,1'-biphenyl]4-carboxylate only: $\delta 168.8,162.4,150.5,149.3,143.1,139.9,139.4,130.7,129.9,128.9,128.8$, $128.6,127.8,127.2,127.1,125.1,120.3,118.5,115.9,110.5$.

HRMS (ESI-TOF) m/z: calcd. for $\left[\mathrm{C}_{25} \mathrm{H}_{18} \mathrm{O}_{3}+\mathrm{Na}\right]^{+} 389.1153$; found 389.1150 .<smiles>Cc1ccccc1OC(=O)c1cccc(C)c1O</smiles>

$o$-Tolyl 2-hydroxy-3-methylbenzoate (15). Prepared according to general procedure using $m$ -cresol $(52 \mu \mathrm{L}, 0.5 \mathrm{mmol})$. The crude product was purified by silica gel chromatography (PE/ $\mathrm{EA}=50: 1)$ to afford the title compound as a light yellow oil (49 $\mathrm{mg}, 81 \%$ yield).

${ }^{1} \mathbf{H}$ NMR $\left(400 \mathrm{MHz}, \mathrm{CDCl}_{3}\right) \delta 10.71(\mathrm{~s}, 1 \mathrm{H}), 7.87(\mathrm{~d}, J=8.0 \mathrm{~Hz}, 1 \mathrm{H}), 7.31(\mathrm{~d}, J=6.5 \mathrm{~Hz}, 1 \mathrm{H})$, $7.23-7.08(\mathrm{~m}, 3 \mathrm{H}), 7.03(\mathrm{~d}, J=8.0 \mathrm{~Hz}, 1 \mathrm{H}), 6.79(\mathrm{t}, J=7.5 \mathrm{~Hz}, 1 \mathrm{H}), 2.21(\mathrm{~s}, 3 \mathrm{H}), 2.14(\mathrm{~s}, 3 \mathrm{H})$. ${ }^{13} \mathbf{C}$ NMR $\left(101 \mathrm{MHz}, \mathrm{CDCl}_{3}\right) \delta 169.1,160.6,148.8,137.2,131.3,130.3,127.8,127.0,126.9$, $126.5,121.9,118.8,110.9,16.1,15.7$.

HRMS (EI) m/z: calcd. for $\left[\mathrm{C}_{15} \mathrm{H}_{14} \mathrm{O}_{3}\right]^{+} 242.0938$; found 242.0942 .<smiles>CCCc1ccccc1OC(=O)c1cccc(C(C)C)c1O</smiles>

2-Isopropylphenyl 2-hydroxy-3-isopropylbenzoate (16). Prepared according to general proc edure using 2-isopropylphenol $(68 \mu \mathrm{L}, 0.5 \mathrm{mmol})$. The crude product was purified by silica ge 1 chromatography $(\mathrm{PE} / \mathrm{EA}=50: 1)$ to afford the title compound as a light yellow oil $(66.6 \mathrm{mg}$, $89 \%$ yield).

${ }^{1} \mathbf{H}$ NMR $\left(300 \mathrm{MHz}, \mathrm{CDCl}_{3}\right) \delta 10.85(\mathrm{~s}, 1 \mathrm{H}), 7.88(\mathrm{dd}, J=8.0,1.5 \mathrm{~Hz}, 1 \mathrm{H}), 7.40(\mathrm{dd}, J=7.5$, $1.5 \mathrm{~Hz}, 1 \mathrm{H}), 7.32-7.27(\mathrm{~m}, 1 \mathrm{H}), 7.20-7.15(\mathrm{~m}, 2 \mathrm{H}), 7.07-6.94(\mathrm{~m}, 1 \mathrm{H}), 6.86(\mathrm{t}, J=8.0 \mathrm{~Hz}$, $1 \mathrm{H}), 3.33$ (hept, $J=7.0 \mathrm{~Hz}, 1 \mathrm{H}), 3.00$ (hept, $J=7.0 \mathrm{~Hz}, 1 \mathrm{H}), 1.19$ (s, 3H), 1.17 (s, 3H), 1.16 (s, $3 \mathrm{H}), 1.13$ (s, 3H).

${ }^{13}$ C NMR $\left(75 \mathrm{MHz}, \mathrm{CDCl}_{3}\right) \delta 169.7,159.9,147.6,140.4,137.2,132.9,127.6,126.9,126.8$, 122.2, 119.1, 111.1, 77.4, 77.0, 76.6, 27.5, 26.6, 23.0, 22.3.

HRMS (EI) m/z: calcd. for $\left[\mathrm{C}_{19} \mathrm{H}_{22} \mathrm{O}_{3}\right]+298.1564$; found 298.1566 .<smiles>COc1ccccc1OC(=O)c1cccc(OC)c1O</smiles>

2-Methoxyphenyl 2-hydroxy-3-methoxybenzoate (17). Prepared according to general proce dure using 3-methoxyphenol $(62.1 \mathrm{mg}, 0.5 \mathrm{mmol})$. The crude product was purified by silica ge 1 chromatography (PE/EA $=5: 1)$ to afford the title compound as a yellow oil $(50 \mathrm{mg}, 73 \%$ yiel d).

${ }^{1} \mathbf{H}$ NMR $\left(300 \mathrm{MHz}, \mathrm{CDCl}_{3}\right) \delta 10.66(\mathrm{~s}, 1 \mathrm{H}), 7.63(\mathrm{dd}, J=8.0,1.5 \mathrm{~Hz}, 1 \mathrm{H}), 7.25-7.13(\mathrm{~m}$, $1 \mathrm{H}), 7.08(\mathrm{~d}, J=6.5 \mathrm{~Hz}, 1 \mathrm{H}), 7.04(\mathrm{dd}, J=8.0,1.5 \mathrm{~Hz}, 1 \mathrm{H}), 6.99-6.87(\mathrm{~m}, 2 \mathrm{H}), 6.83(\mathrm{t}, J=$ $8.0 \mathrm{~Hz}, 1 \mathrm{H}), 3.85$ (s, 3H), 3.74 (s, 3H). 
${ }^{13} \mathbf{C}$ NMR $\left(75 \mathrm{MHz}, \mathrm{CDCl}_{3}\right) \delta 168.7,152.4,151.1,148.6,139.0,127.4,122.7,121.6,120.8$, 118.7, 117.1, 112.5, 112.0, 56.2, 55.8.

HRMS (ESI-TOF) m/z: calcd. for $\left[\mathrm{C}_{15} \mathrm{H}_{14} \mathrm{O}_{5}+\mathrm{Na}\right]^{+} 297.0739$; found 297.0742 .<smiles>O=C(Oc1ccccc1-c1ccccc1)c1cccc(-c2ccccc2)c1O</smiles>

[1,1'-Biphenyl]-2-yl 2-hydroxy-[1,1'-biphenyl]-3-carboxylate (18). Prepared according to g eneral procedure using [1,1'-biphenyl]-2-ol $(62.1 \mathrm{mg}, 0.5 \mathrm{mmol})$. The crude product was purifi ed by silica gel chromatography (PE/EA $=50: 1)$ to afford the title compound as a yellow oil (8 $5.1 \mathrm{mg}, 75 \%$ yield).

${ }^{1} \mathbf{H}$ NMR $\left(300 \mathrm{MHz}, \mathrm{CDCl}_{3}\right) \delta 10.93(\mathrm{~s}, 1 \mathrm{H}), 7.99(\mathrm{dd}, J=8.0,1.5 \mathrm{~Hz}, 1 \mathrm{H}), 7.65-7.33(\mathrm{~m}$, $15 \mathrm{H}), 7.02(\mathrm{t}, J=9.0 \mathrm{~Hz}, 1 \mathrm{H})$.

${ }^{13} \mathrm{C}$ NMR $\left(75 \mathrm{MHz}, \mathrm{CDCl}_{3}\right) \delta 169.0,159.1,146.9,137.0,136.8,136.7,134.8,131.0,130.3$, 129.4, 129.1, 128.7, 128.4, 128.2, 128.0, 127.5, 127.3, 126.7, 122.7, 119.1, 111.7.

HRMS (ESI-TOF) m/z: calcd. for $\left[\mathrm{C}_{25} \mathrm{H}_{18} \mathrm{O}_{3}+\mathrm{Na}\right]+389.1153$; found 389.1148 . 


\section{Spectral Date of the Products}

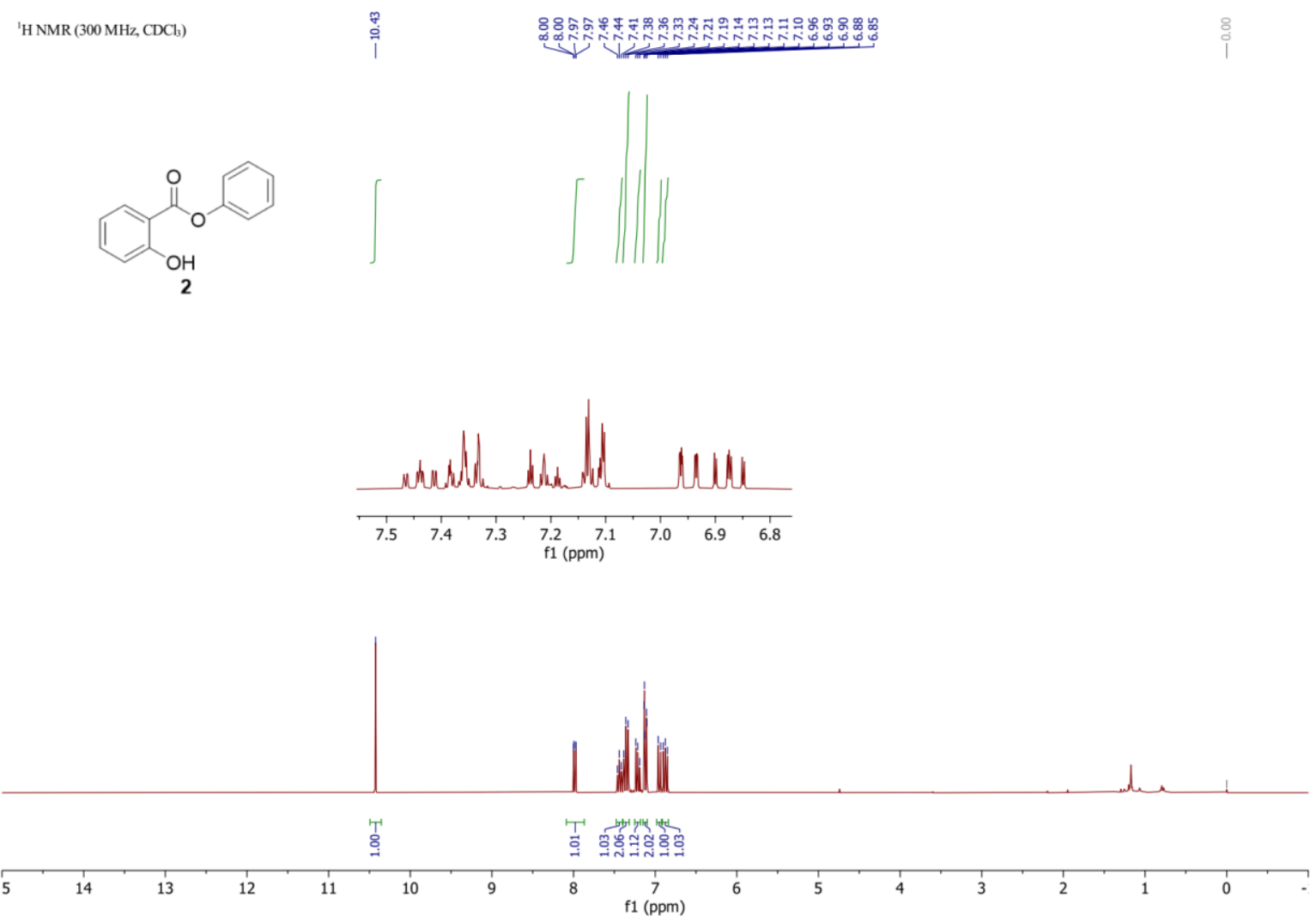

${ }^{13} \mathrm{CNMR}\left(75 \mathrm{MHz}, \mathrm{CDCl}_{3}\right)$
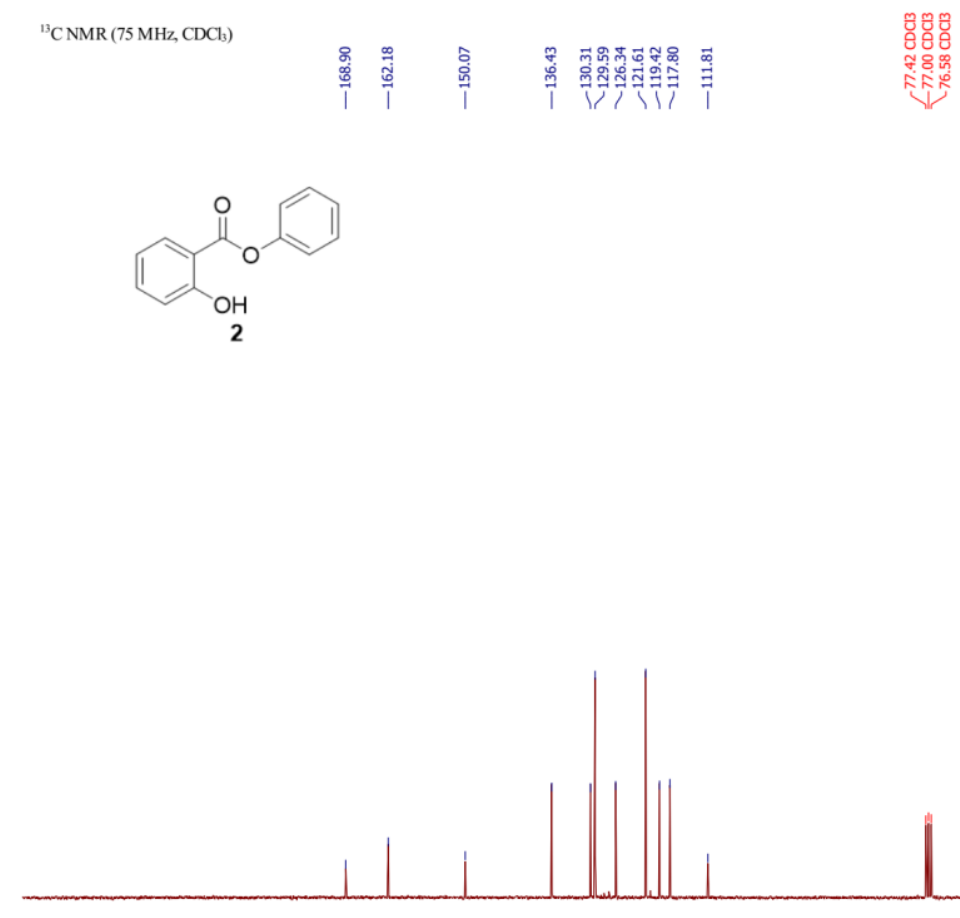

$\begin{array}{lllllllllllllllllllllllllllllllllll}1 & 1 & 200 & 190 & 180 & 170 & 160 & 150 & 140 & 130 & 120 & 110 & 100 & 90 & 80 & 70 & 60 & 50 & 40 & 30 & 20 & 10 & 0 & -10 & -2\end{array}$ 
'H NMR (400 MHz, CDCls)

(1)

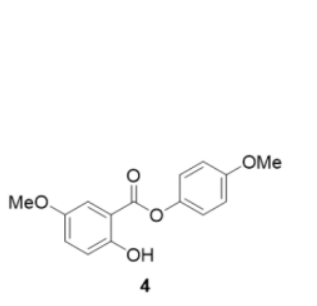

势
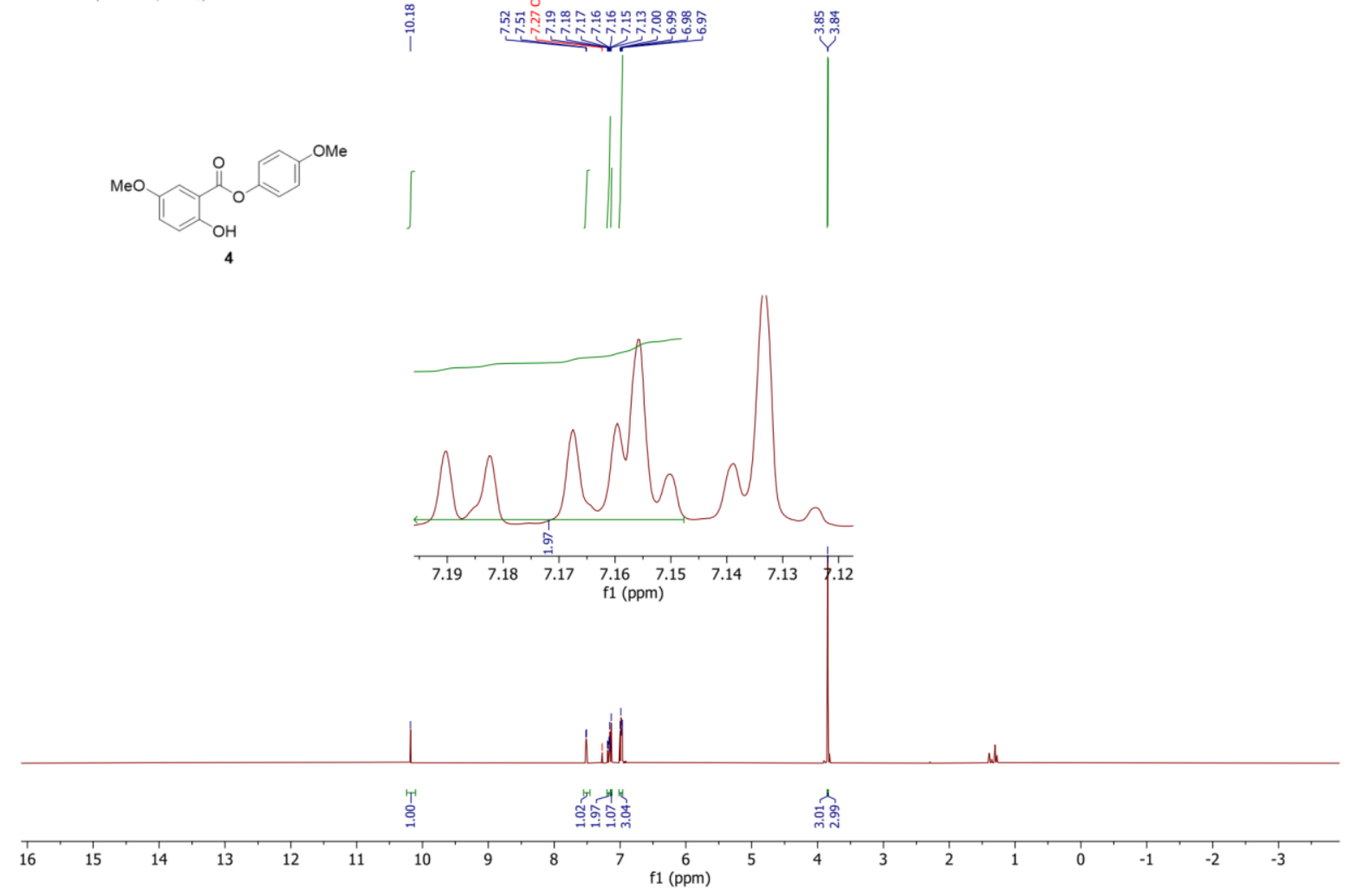

${ }^{13} \mathrm{CNMR}\left(101 \mathrm{MHz}, \mathrm{CDCl}_{3}\right)$

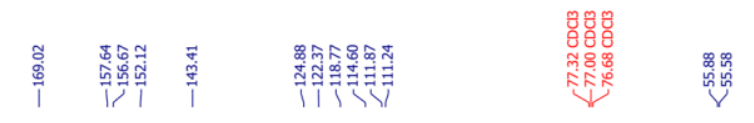
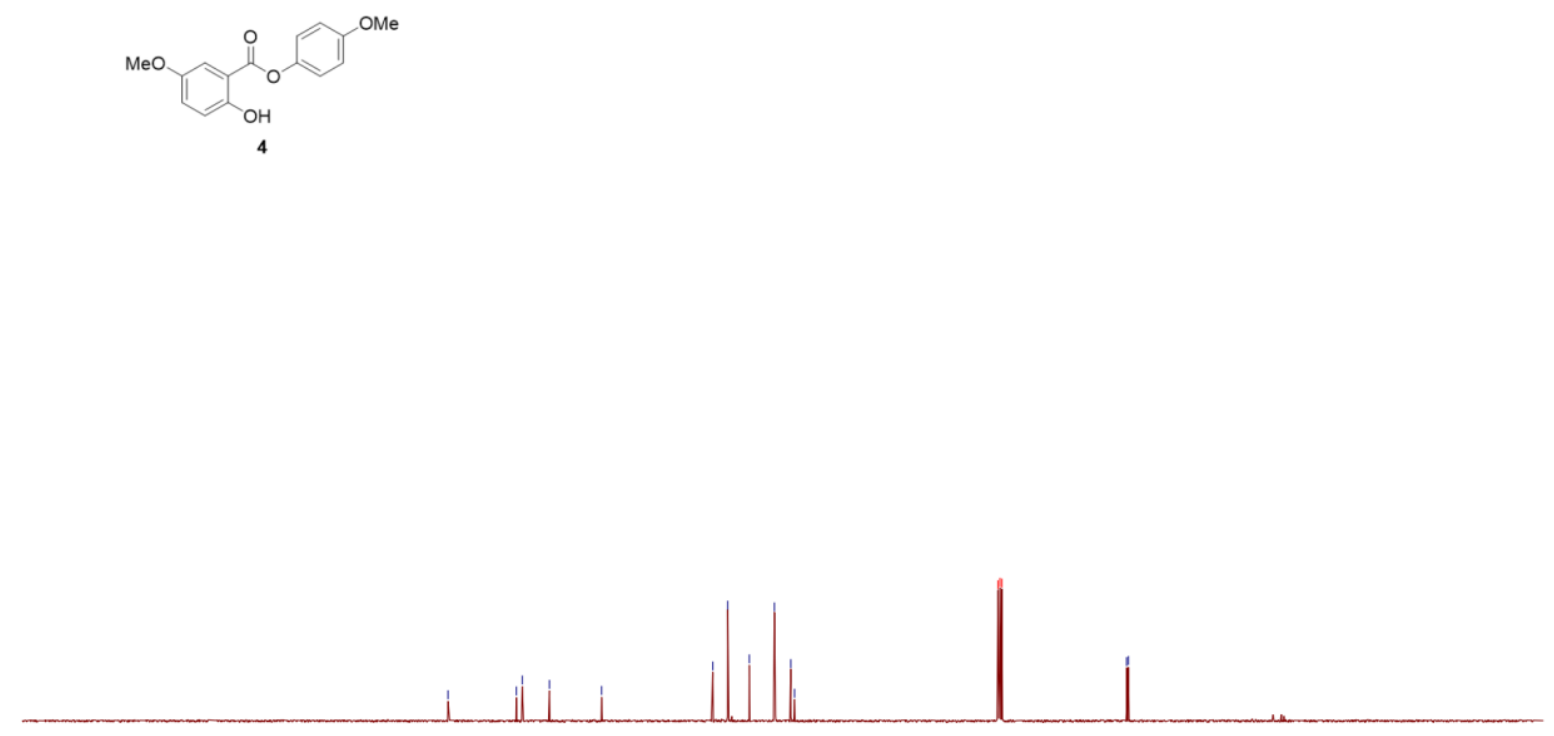

$\begin{array}{llllllllllllllllllllllllllllllllll}1 & 1 \\ 230 & 220 & 210 & 200 & 190 & 180 & 170 & 160 & 150 & 140 & 130 & 120 & 110 & 100 & 90 & 80 & 70 & 60 & 50 & 40 & 30 & 20 & 10 & 0 & -10\end{array}$ 


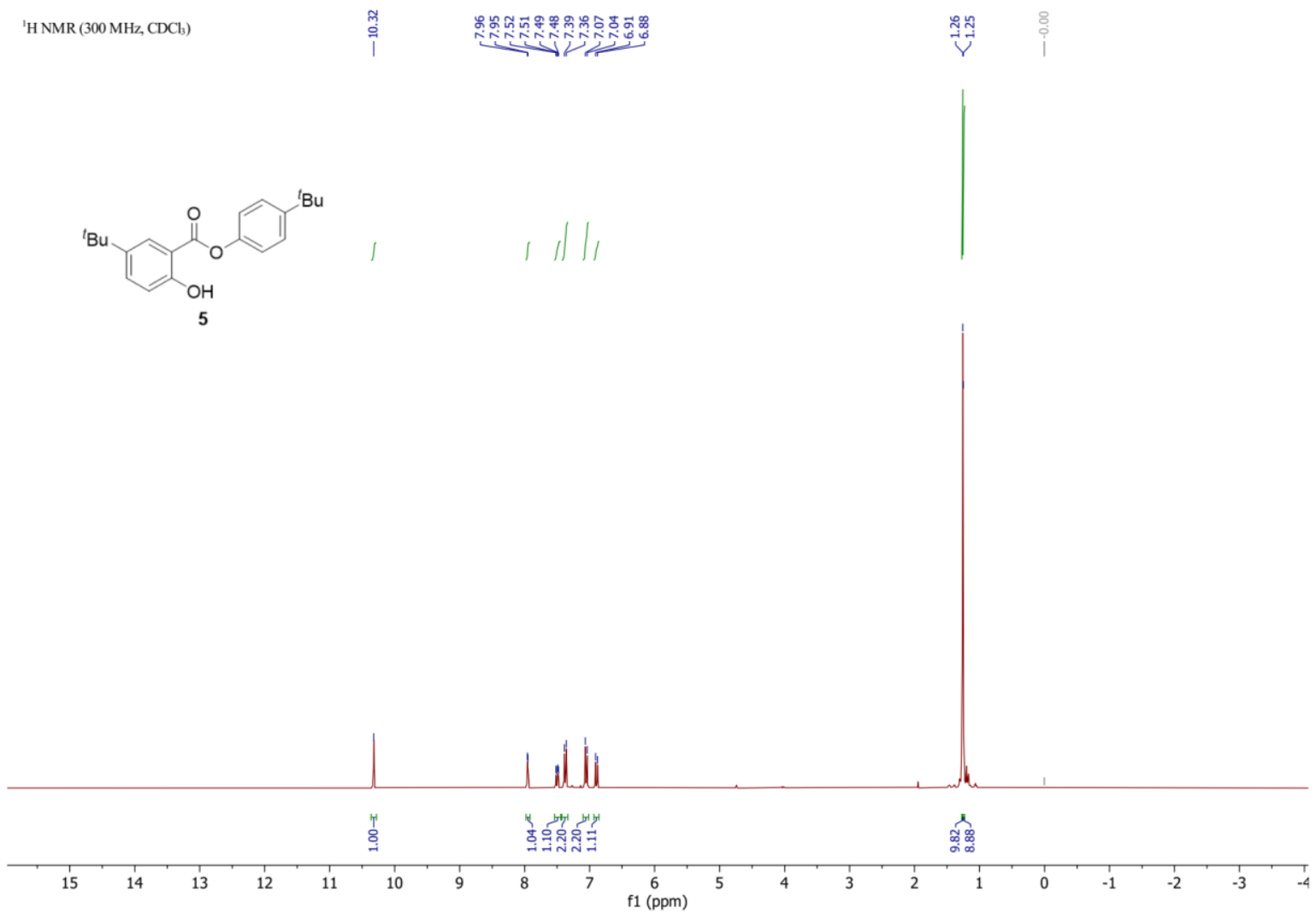

${ }^{13} \mathrm{CNMR}\left(75 \mathrm{MHz}, \mathrm{CDCl}_{3}\right)$

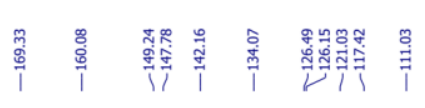

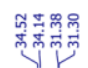
$\underbrace{O H}_{5}$

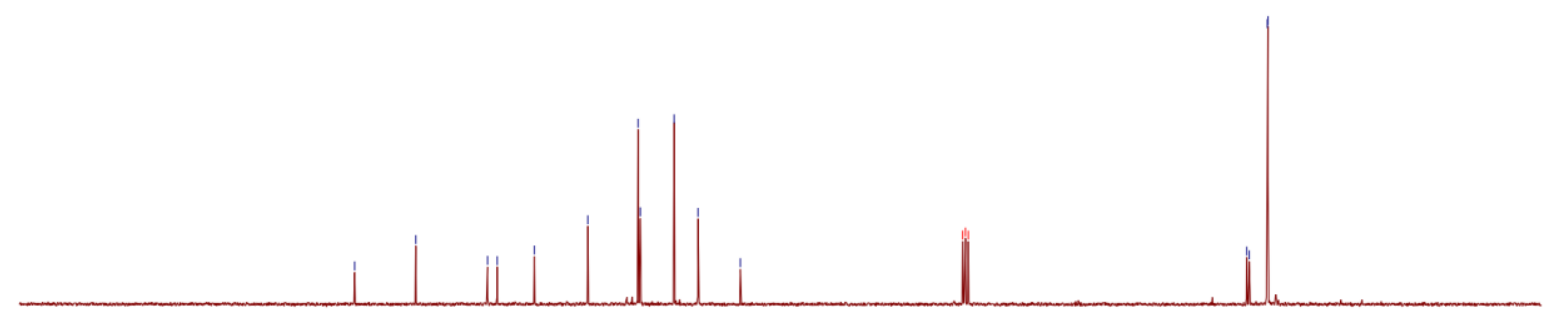

\begin{tabular}{lllllllllllllllllllllllllllll}
\hline 20 & 210 & 200 & 190 & 180 & 170 & 160 & 150 & 140 & 130 & 120 & 110 & 100 & 90 & 80 & 70 & 60 & 50 & 40 & 30 & 20 & 10 & 0 & -1
\end{tabular} 


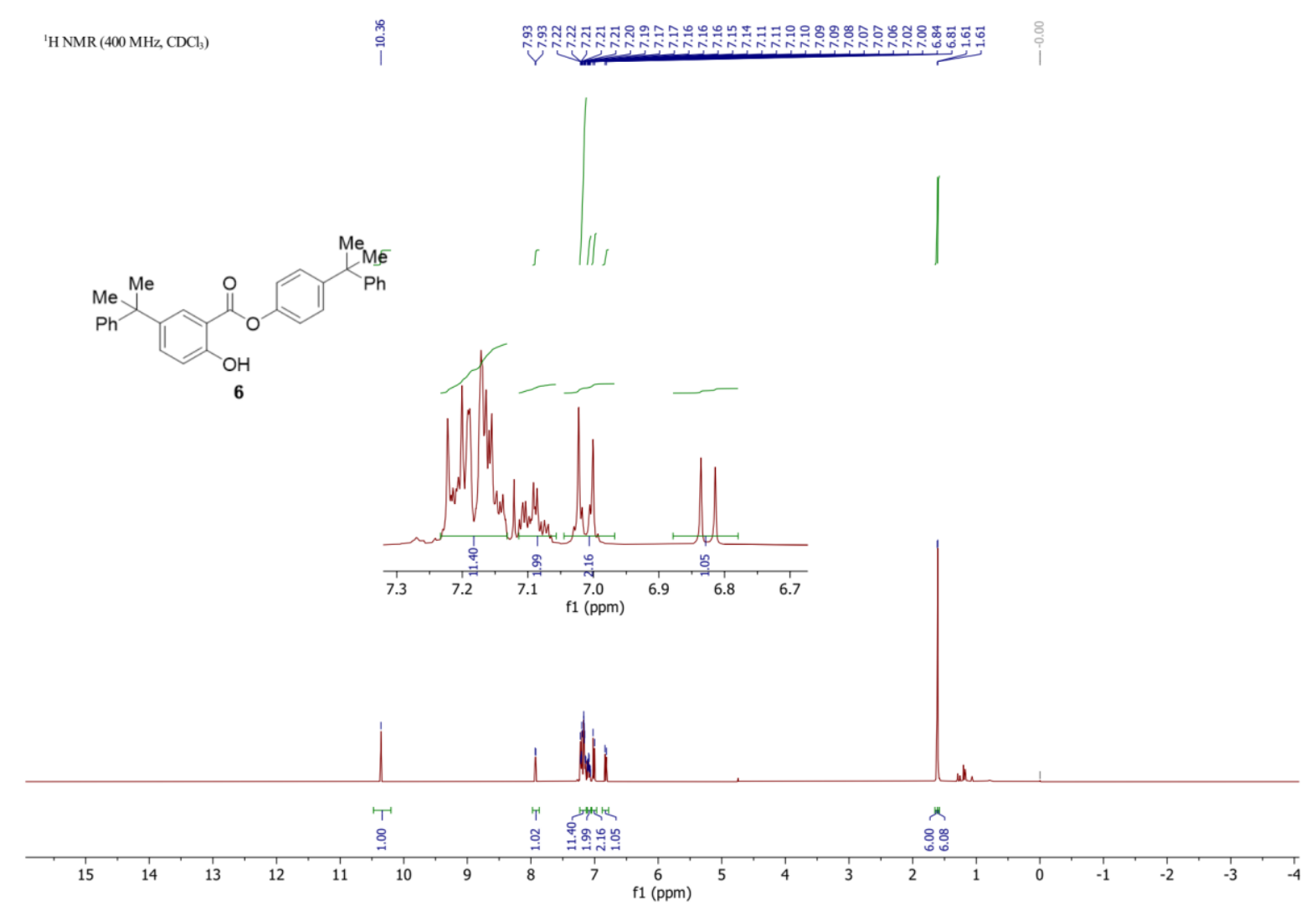

${ }^{13} \mathrm{CNMR}\left(101 \mathrm{MHz}, \mathrm{CDCl}_{3}\right)$
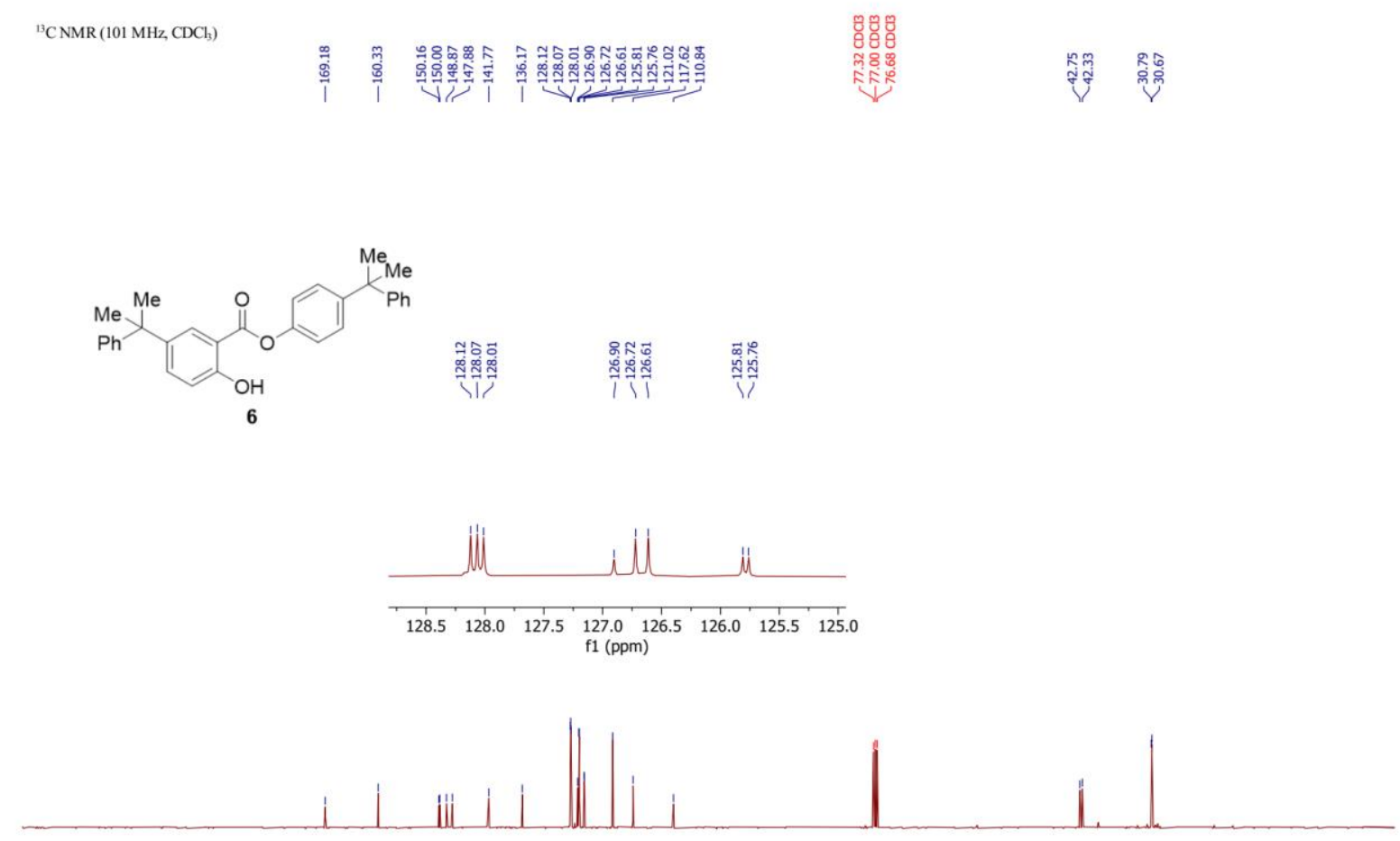

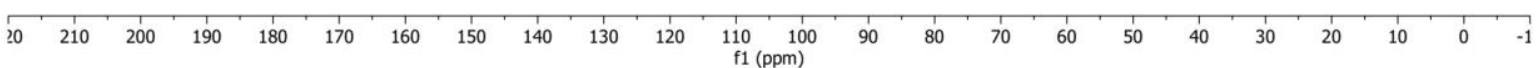



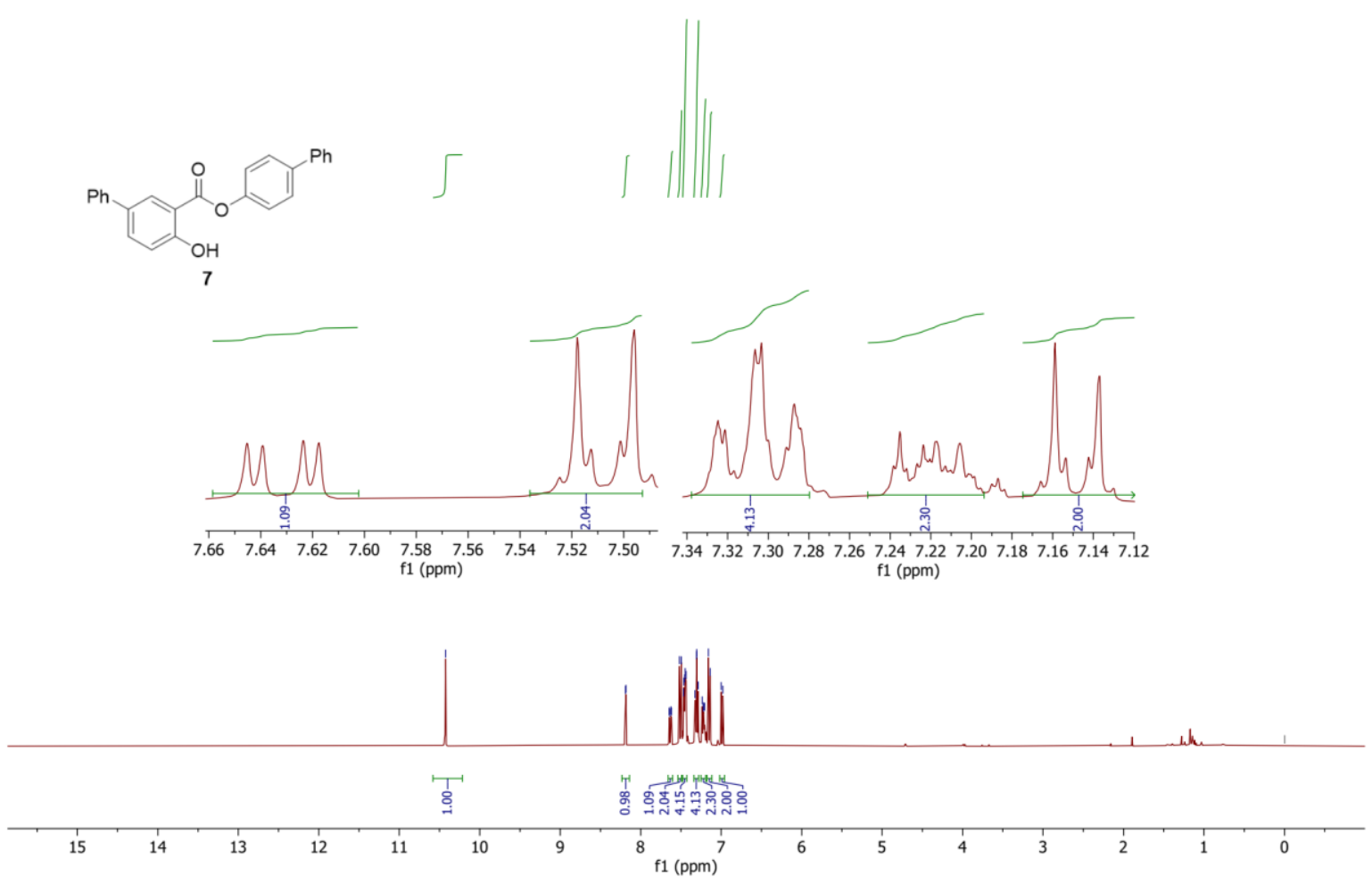

${ }^{13} \mathrm{CNMR}\left(75 \mathrm{MHz}, \mathrm{CDCl}_{3}\right)$
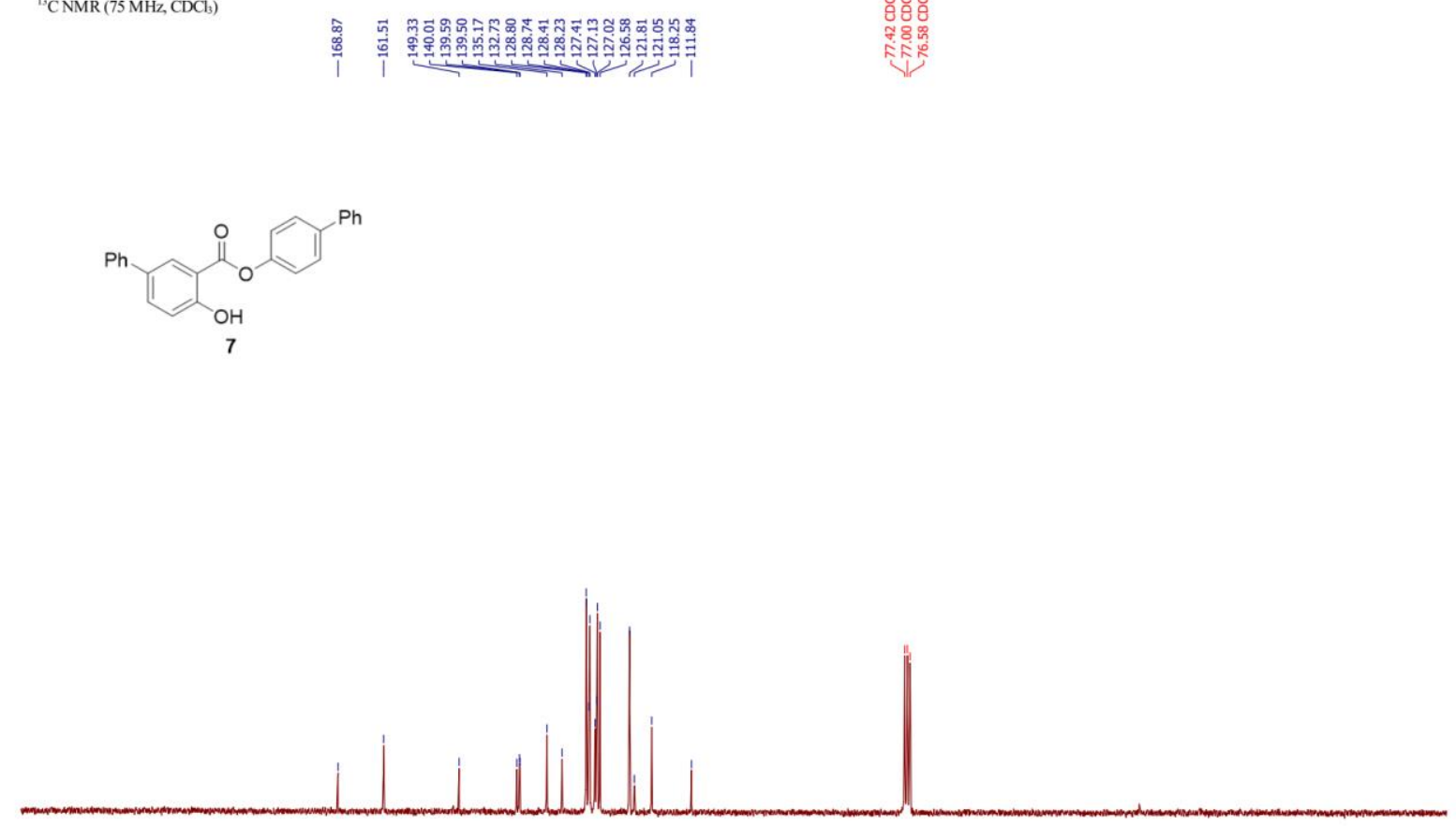

$\begin{array}{lllllllllllllllllllllllllllll}20 & 210 & 200 & 190 & 180 & 170 & 160 & 150 & 140 & 130 & 120 & 110 & 100 & 90 & 80 & 70 & 60 & 50 & 40 & 30 & 20 & 10 & 0 & -1\end{array}$ 
<smiles>CCc1ccc(OC(=O)c2cc(CC)ccc2O)cc1</smiles>

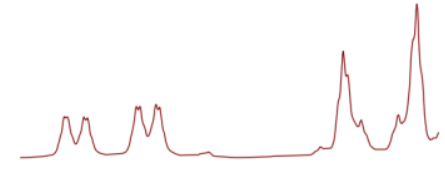

$\begin{array}{llllllllllllll}7.46 & 7.44 & 7.42 & 7.40 & 7.38 & 7.36 & 7.34 & 7.32 & 7.30\end{array}$ f1 (ppm)
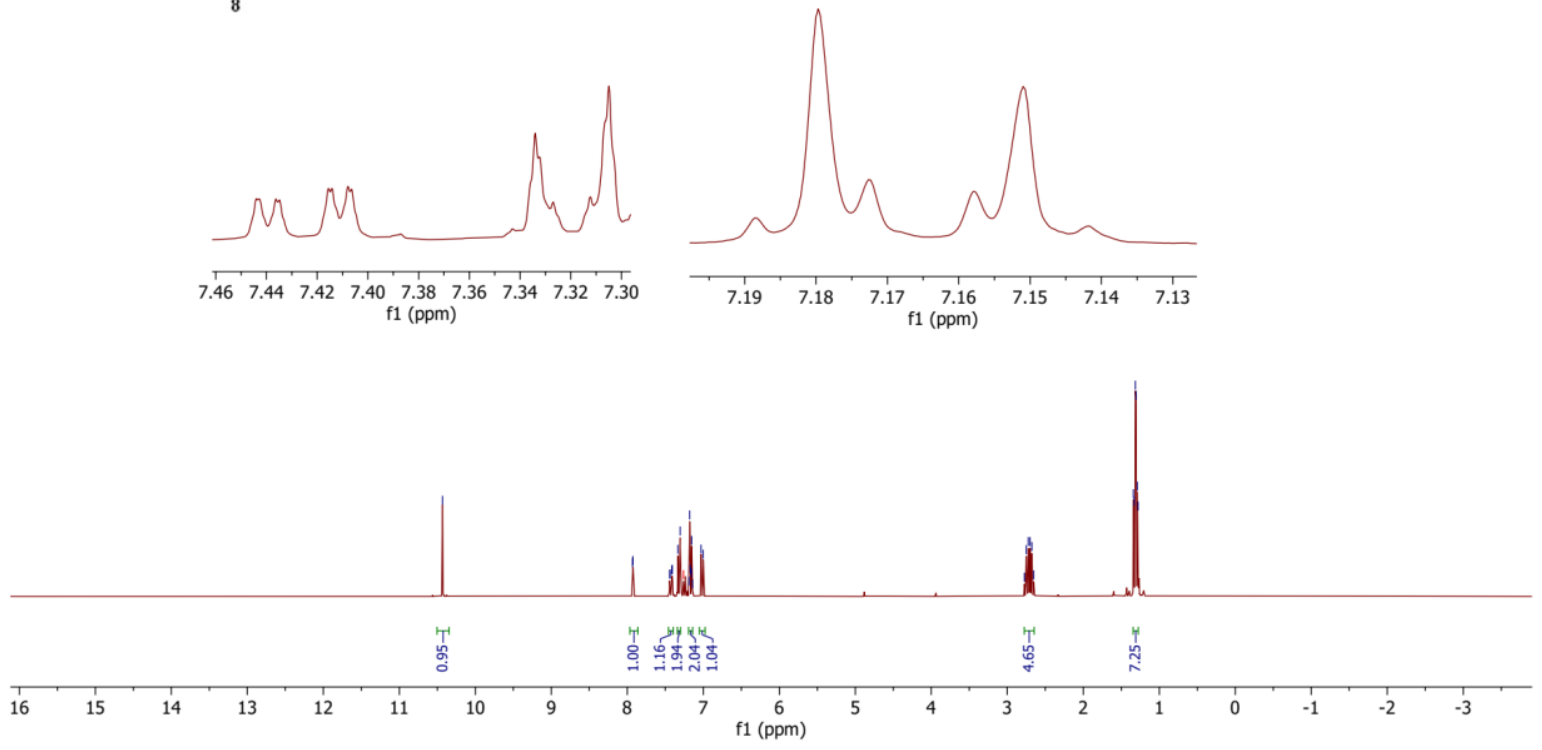

${ }^{13} \mathrm{CNMR}\left(75 \mathrm{MHz}, \mathrm{CDCl}_{3}\right)$
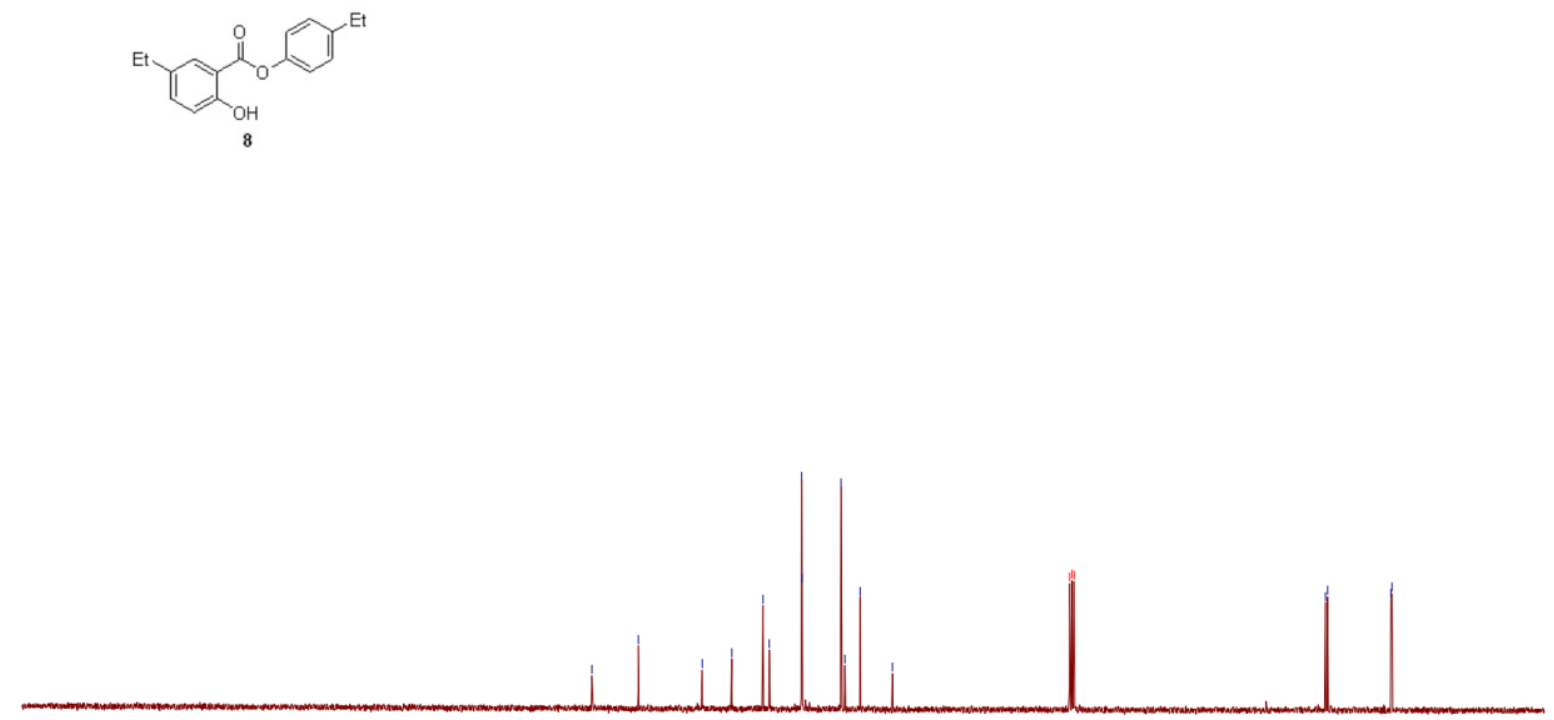

$\begin{array}{llllllllllllllllllllllllllllllllllllllllllllllllll}270 & 260 & 250 & 240 & 230 & 220 & 210 & 200 & 190 & 180 & 170 & 160 & 150 & 140 & 130 & 120 & 110 & 100 & 90 & 80 & 70 & 60 & 50 & 40 & 30 & 20 & 10 & 0 & -10\end{array}$ 
'H NMR (300 MHz, CDCl3)<smiles>Cc1ccc(OC(=O)c2cc(C)ccc2O)cc1</smiles>
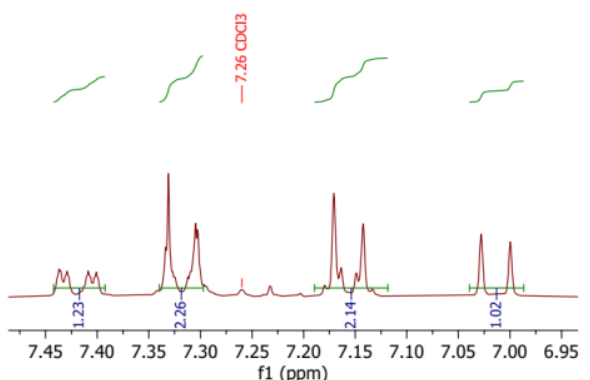

f1 (ppm)

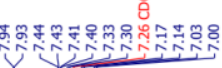

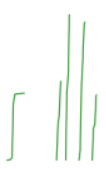

ididi
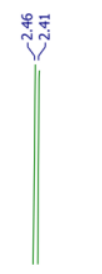
<smiles>C#CCCCCCCCCCCC(C)=O</smiles>

10

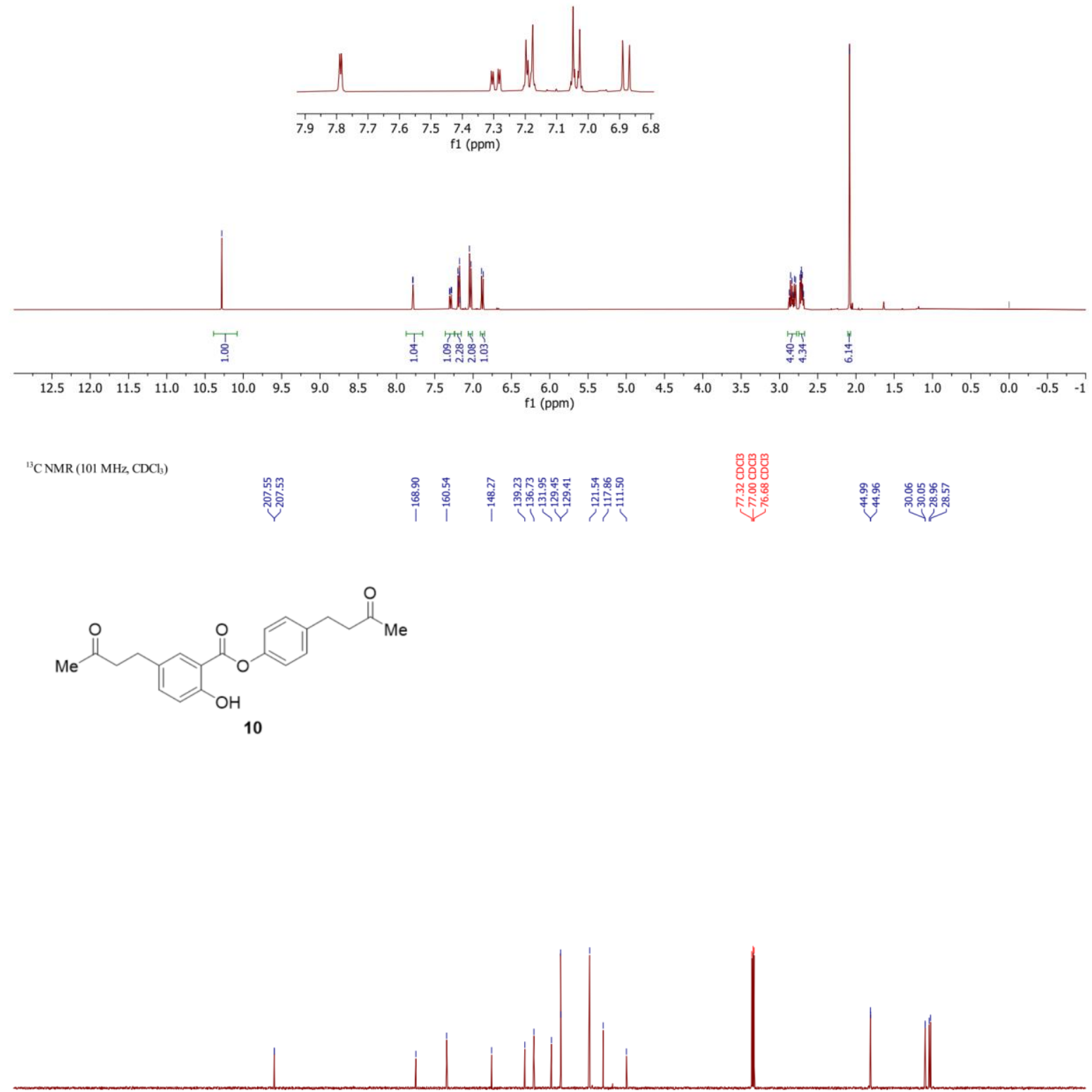

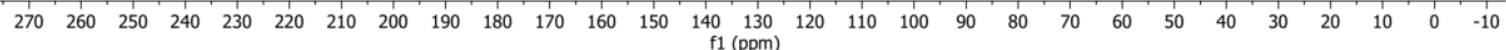




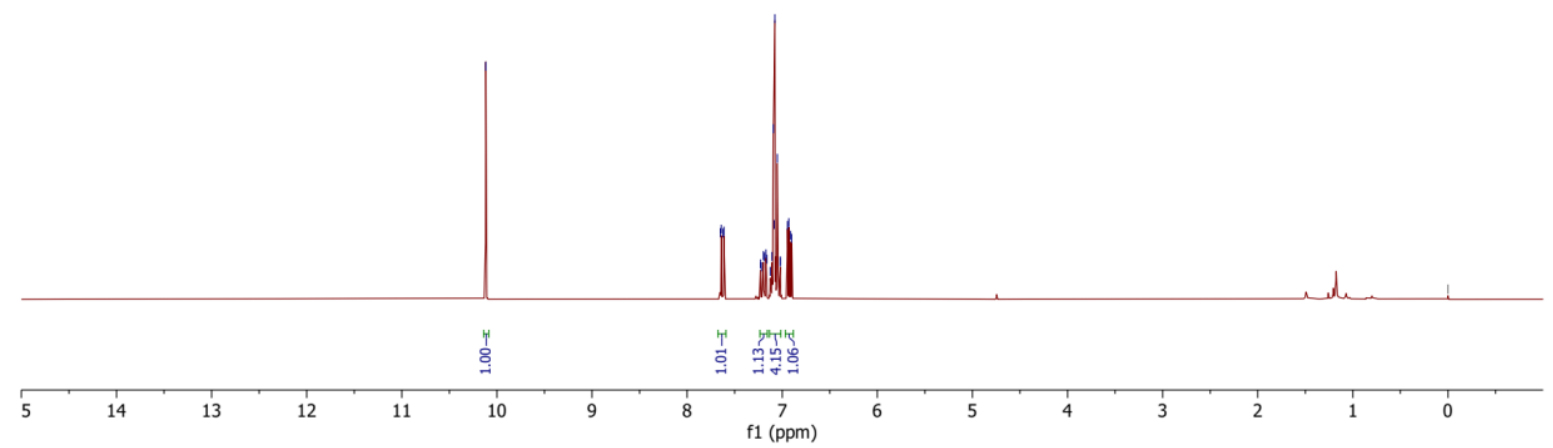

${ }^{13} \mathrm{CNMR}\left(75 \mathrm{MHz}, \mathrm{CDCl}_{3}\right)$
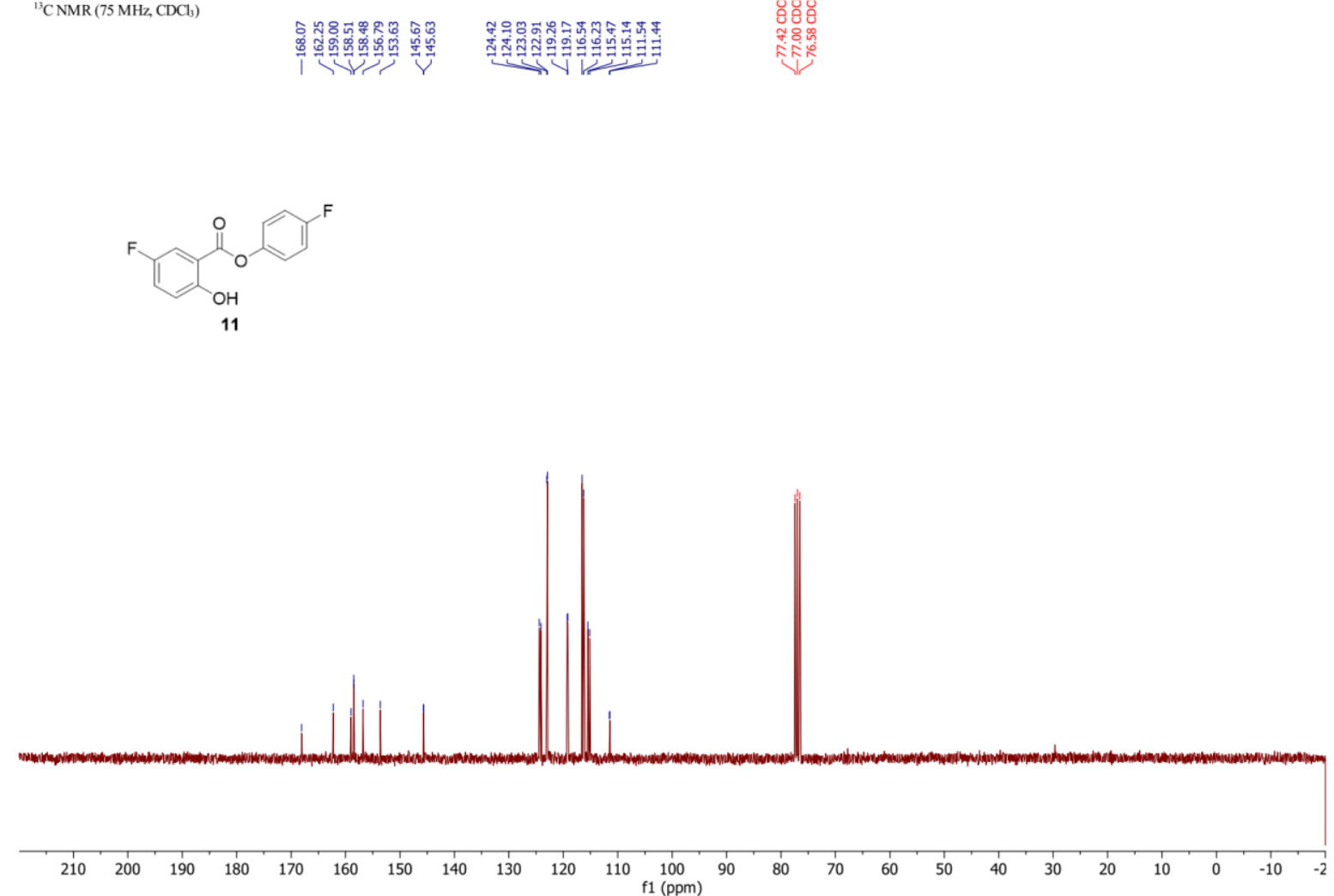
${ }^{19} \mathrm{~F} \mathrm{NMR}\left(282 \mathrm{MHz}, \mathrm{CDCl}_{3}\right)$
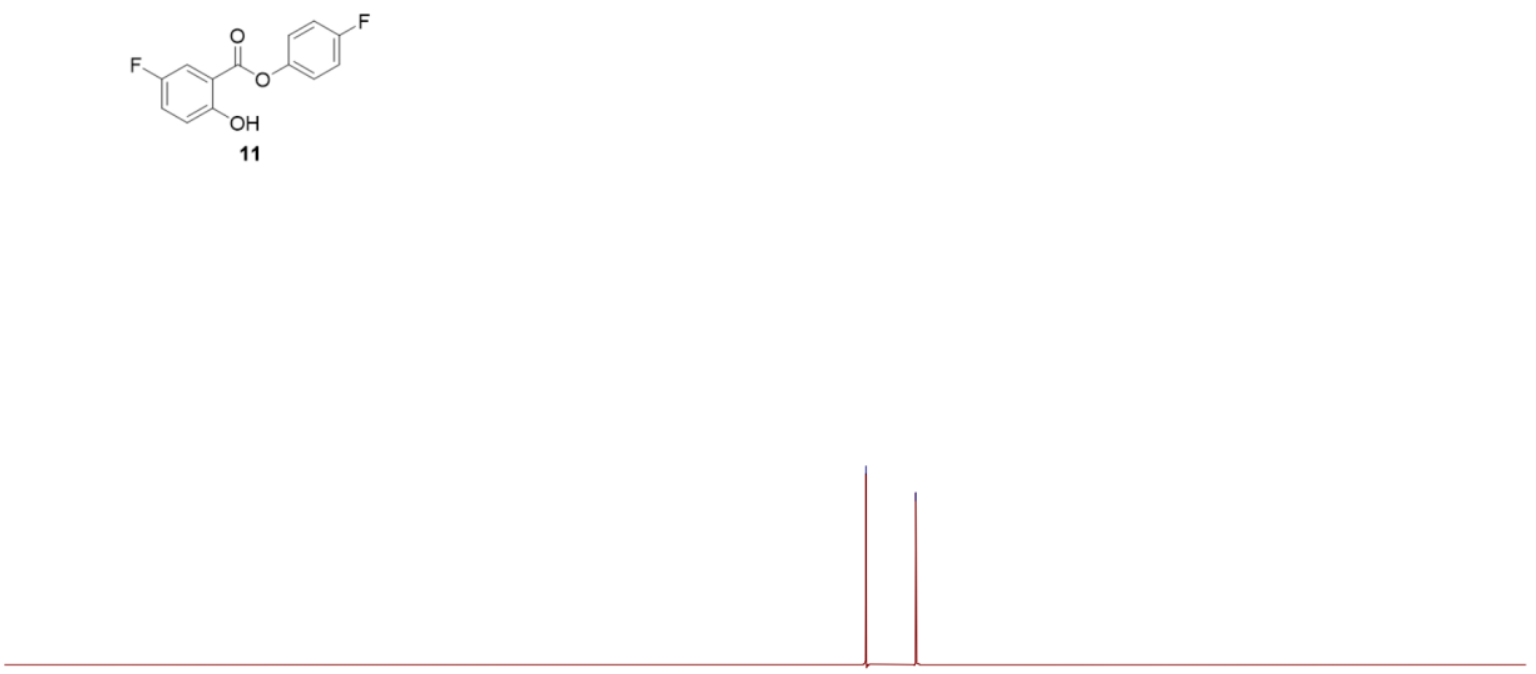

$\begin{array}{llllllllllllllllllllllll}1 & 10 & 0 & -10 & -20 & -30 & -40 & -50 & -60 & -70 & -80 & -90 & -100 & -110 & -120 & -130 & -140 & -150 & -160 & -170 & -180 & -190 & -200 & -210\end{array}$ 

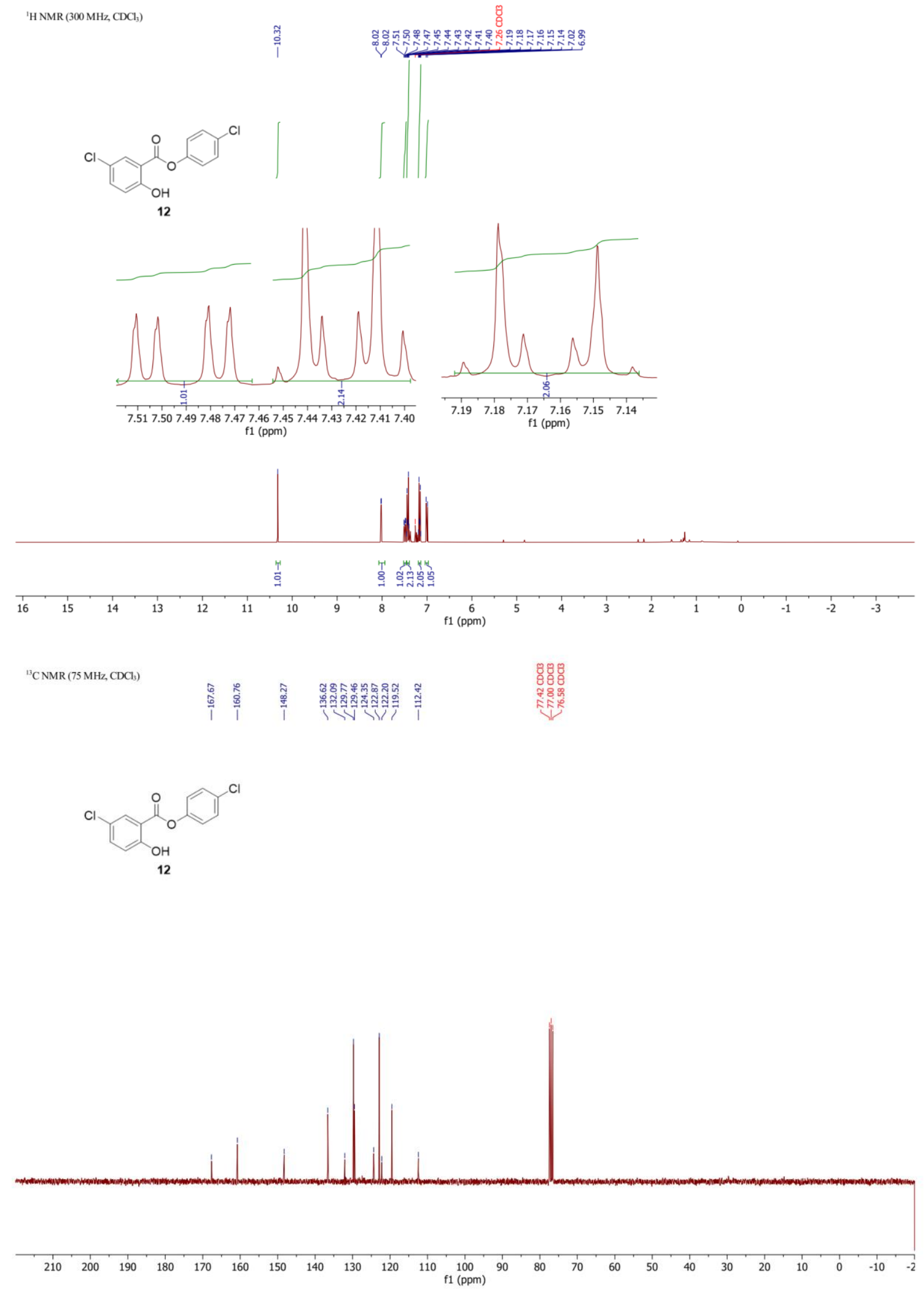
${ }^{2} \mathrm{H} \mathrm{NMR}\left(300 \mathrm{MHz}, \mathrm{CDCl}_{3}\right.$ ) integration of $p$-Tolyl 2-hydroxy-4-methylbenzoate AND $p$ -

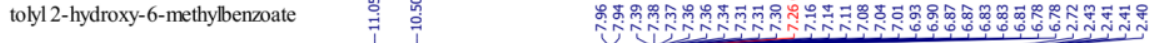
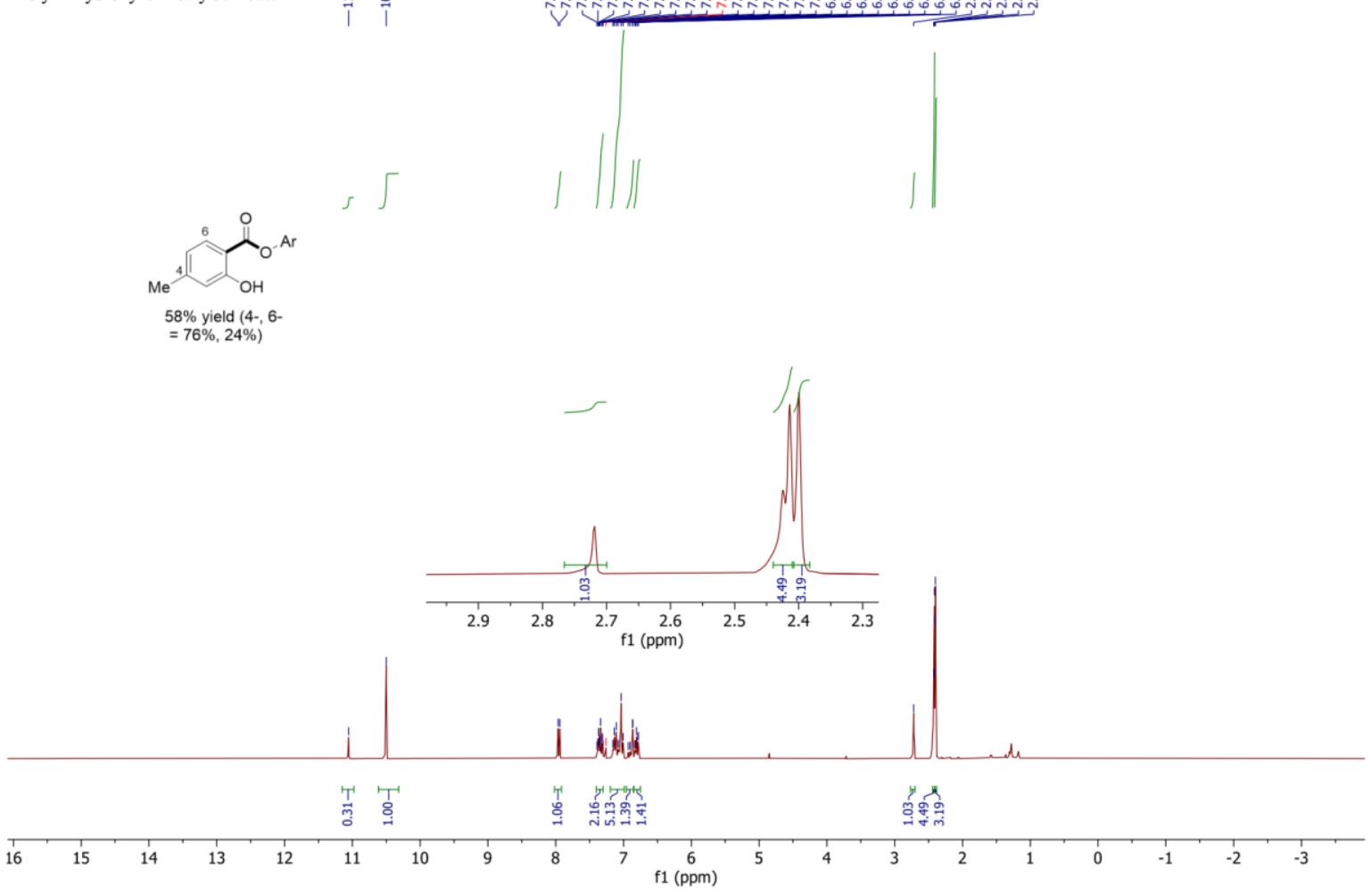

${ }^{13} \mathrm{CNMR}\left(75 \mathrm{MHz}, \mathrm{CDCl}_{3}\right)$ peak picking of $p$-Tolyl 2-hydroxy-4-methylbenzoate only
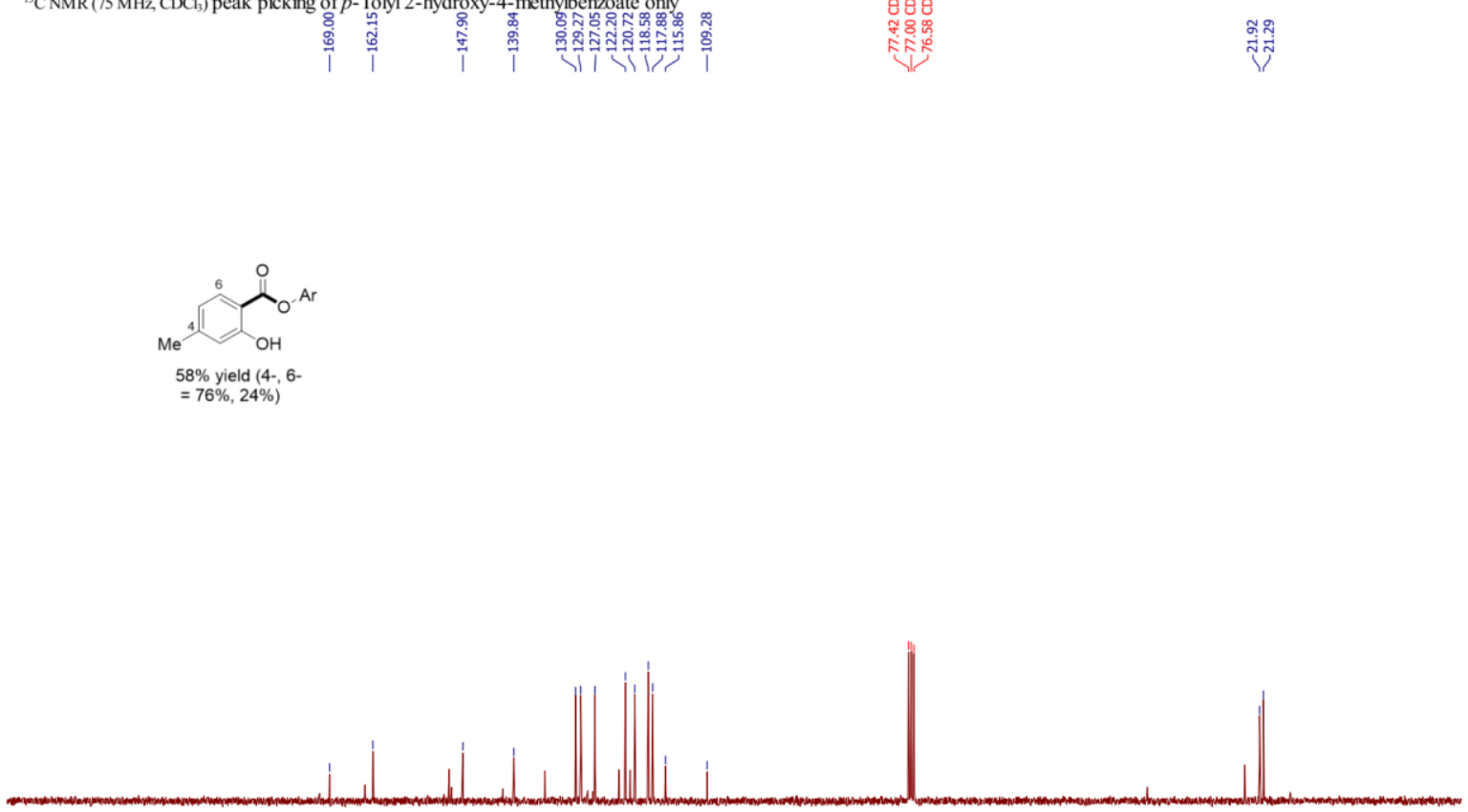

\begin{tabular}{lllllllllllllllllllllllllllll}
\hline 20 & 210 & 200 & 190 & 180 & 170 & 160 & 150 & 140 & 130 & 120 & 110 & 100 & 90 & 80 & 70 & 60 & 50 & 40 & 30 & 20 & 10 & 0
\end{tabular} 


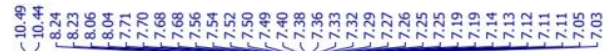

'H NMR (400 MHz, CDCl 3 )integration of [1,1'-Biphenyl]-4-yl 3-hydroxy-[1,1'-

biphenyl]-4-carboxylate AND [1,1'-biphenyl]-4-yl 3-hydroxy-[1,1'-biphenyl]-2-

carboxylate

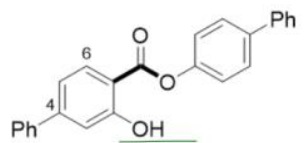

$14,74 \%$ yield $(4-, 6-=92 \%, 8 \%)$

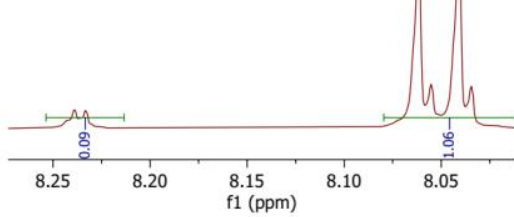

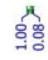

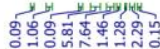
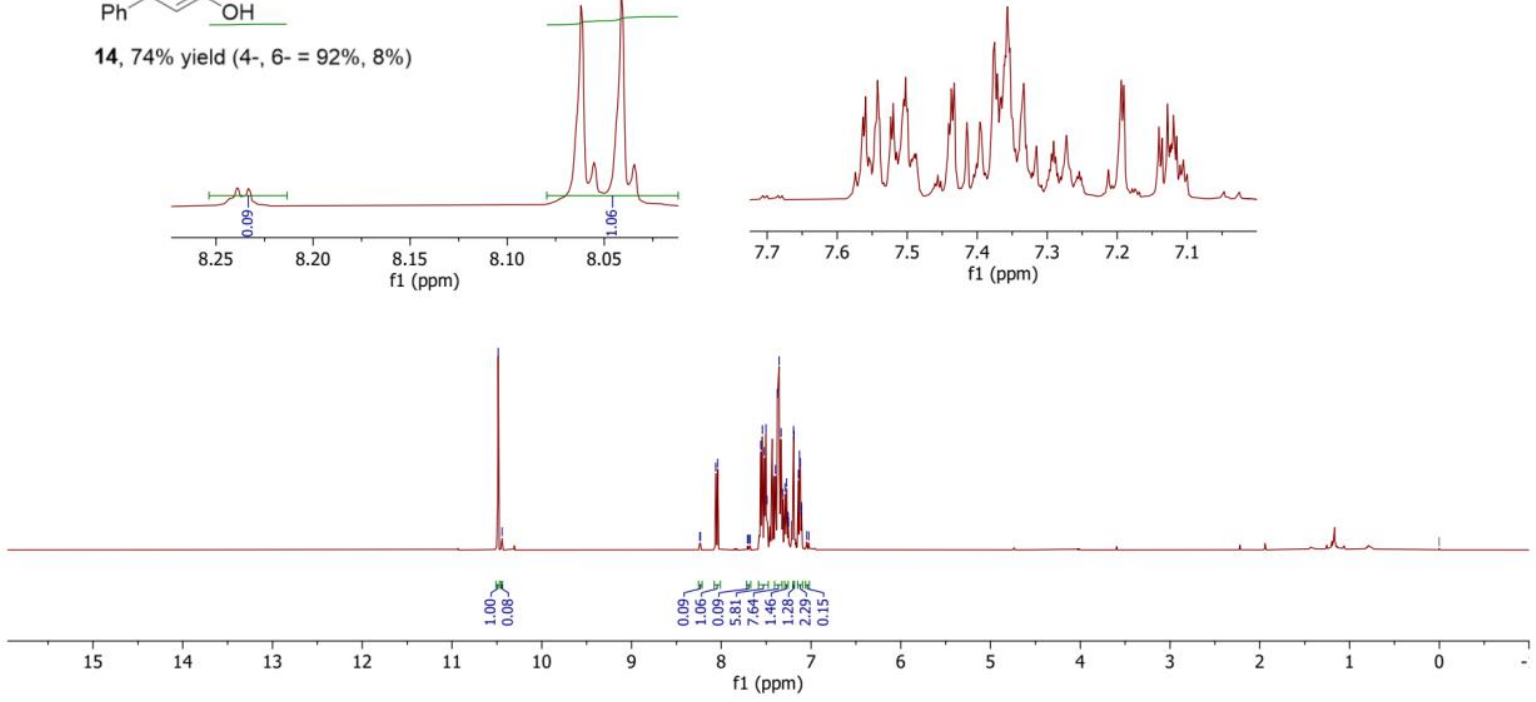

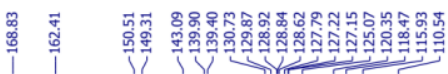

${ }^{13} \mathrm{C} \mathrm{NMR}(101 \mathrm{MHz}, \mathrm{CDCl})$ peak picking of [1,1'-Biphenyl]-4-yl 3-hydroxy-[1,1'-

biphenyl]-4-carboxylate only<smiles>O=C(Oc1ccc(-c2ccccc2)cc1)c1ccc(-c2ccccc2)cc1O</smiles>

$14,74 \%$ yield $(4-, 6-=92 \%, 8 \%)$

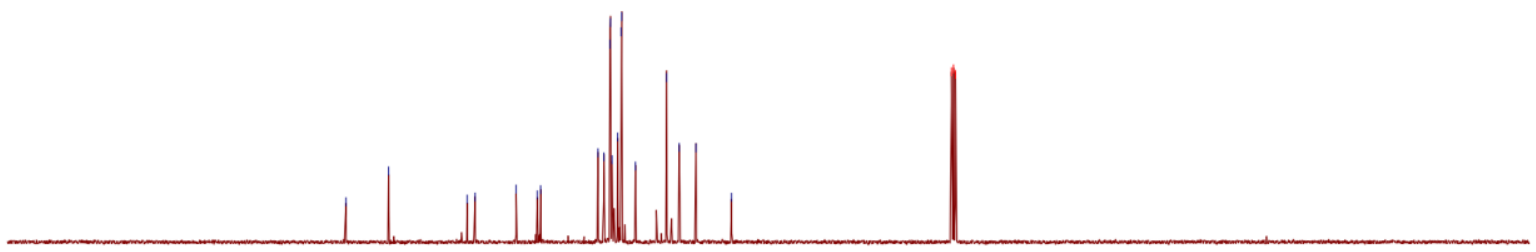

$\begin{array}{lllllllllllllllllllllllllllllllllllllll}20 & 210 & 200 & 190 & 180 & 170 & 160 & 150 & 140 & 130 & 120 & 110 & 100 & 90 & 80 & 70 & 60 & 50 & 40 & 30 & 20 & 10 & 0 & -1\end{array}$ 

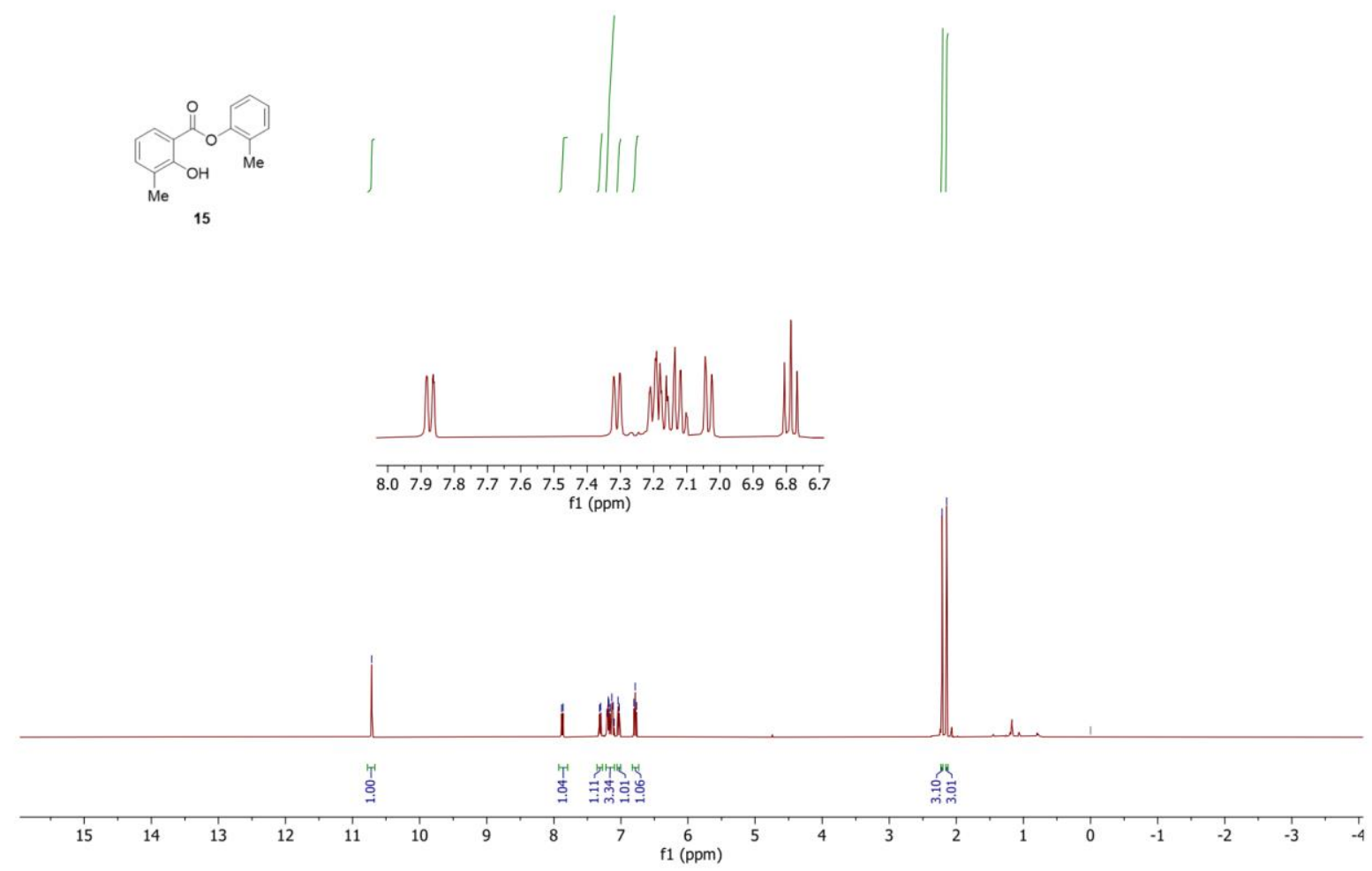

${ }^{13} \mathrm{CNMR}\left(101 \mathrm{MHz}, \mathrm{CDCl}_{3}\right)$
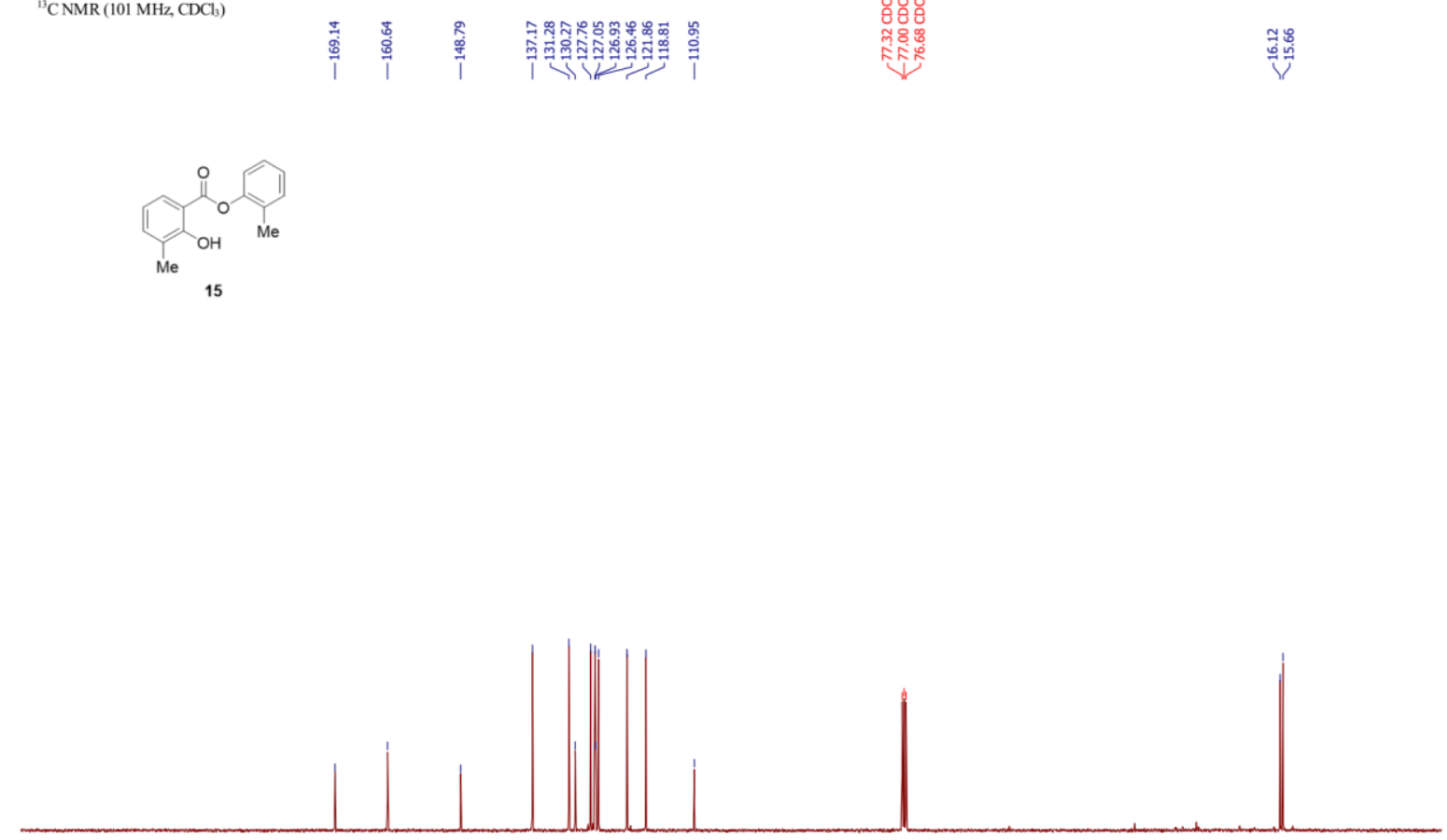

$\begin{array}{llllllllllllllllllllllllllll}20 & 210 & 200 & 190 & 180 & 170 & 160 & 150 & 140 & 130 & 120 & 110 & 100 & 90 & 80 & 70 & 60 & 50 & 40 & 30 & 20 & 10 & 0 & -1\end{array}$ 


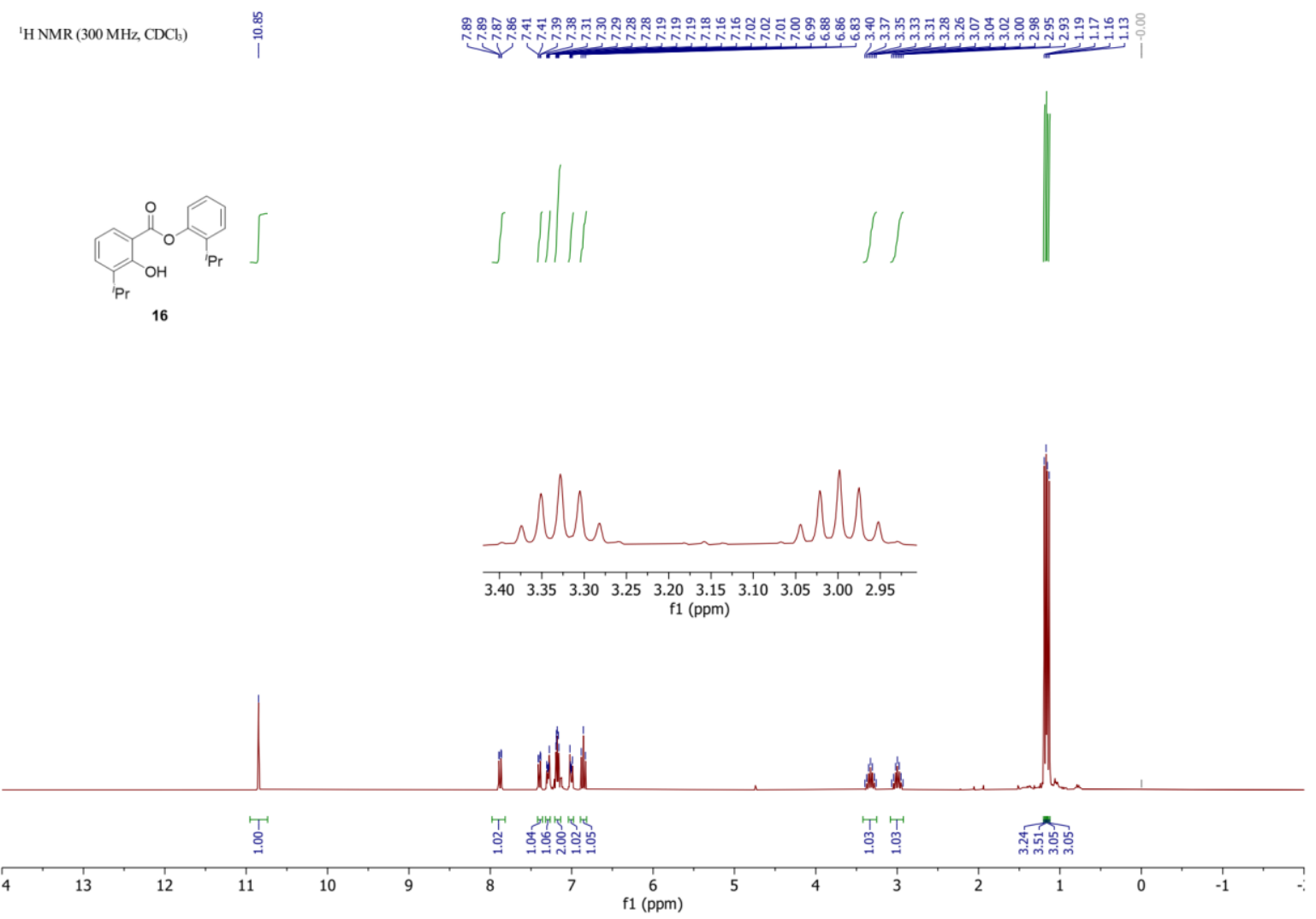

${ }^{13} \mathrm{CNMR}\left(75 \mathrm{MHz}, \mathrm{CDCl}_{3}\right)$

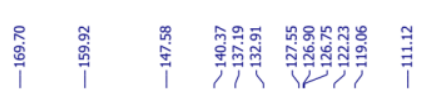<smiles>CCCc1ccccc1OC(=O)c1cccc(CC)c1O</smiles>

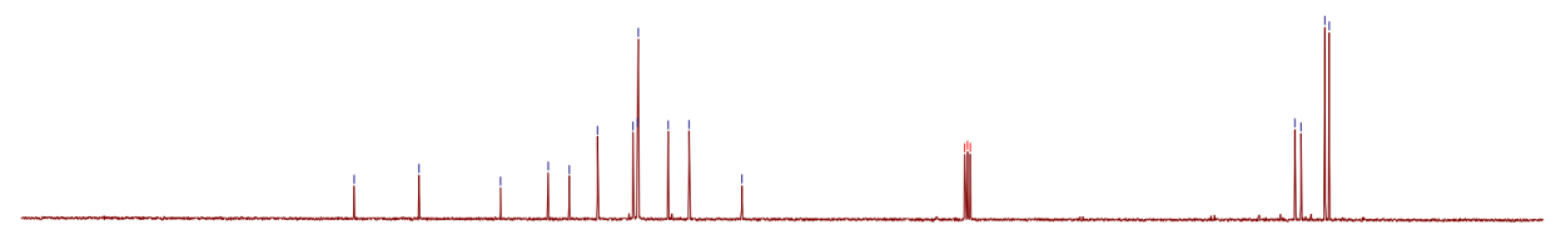

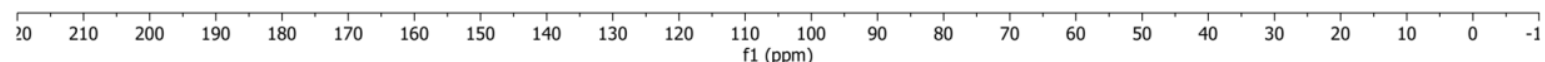




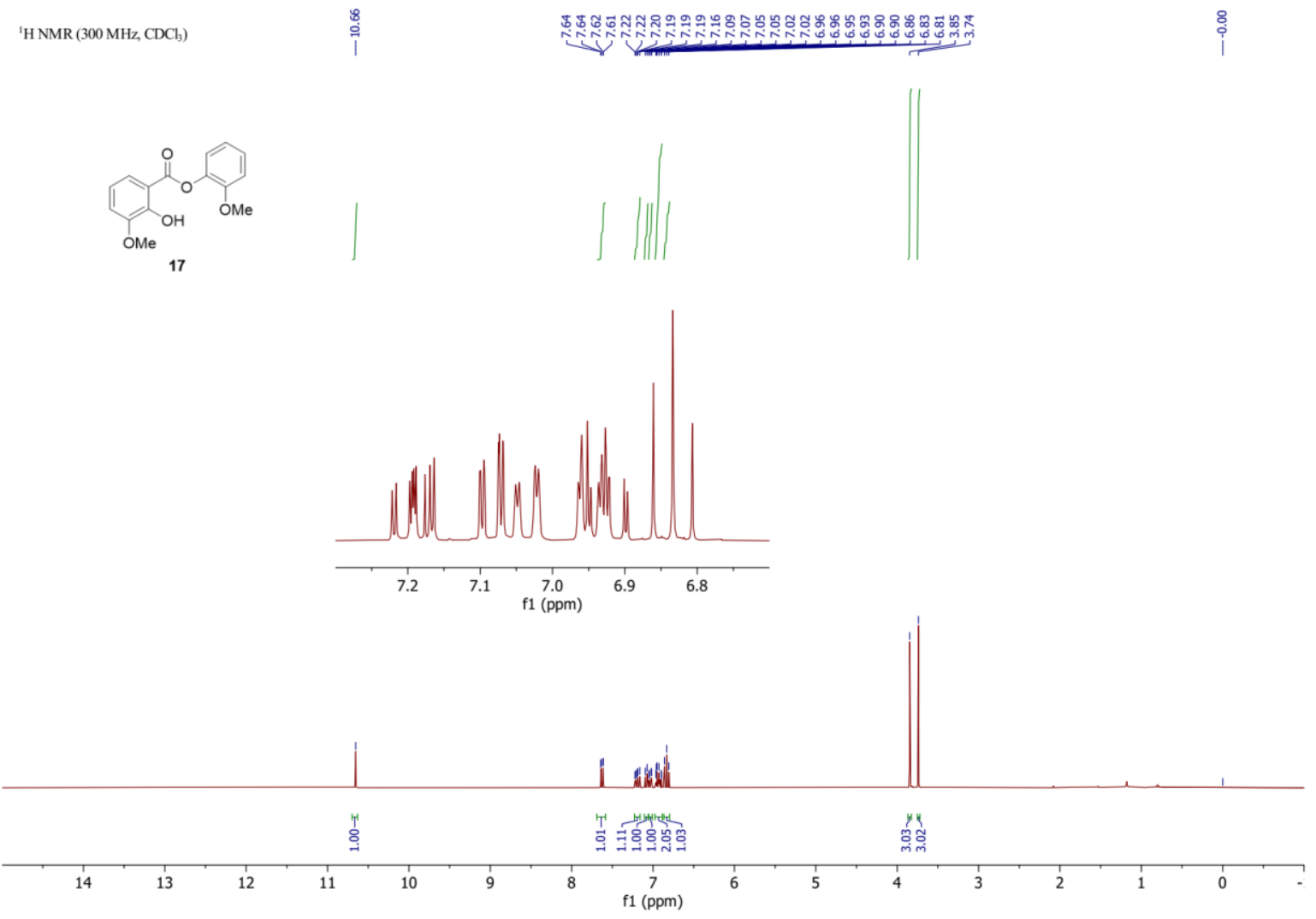

${ }^{13} \mathrm{CNMR}\left(75 \mathrm{MHz}, \mathrm{CDCl}_{3}\right)$

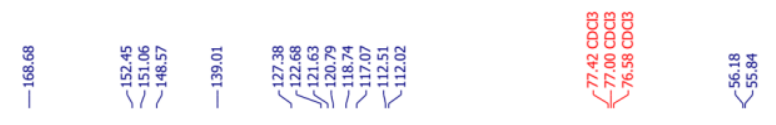

$$
\mathrm{OMe}_{17}^{\mathrm{OH}}
$$

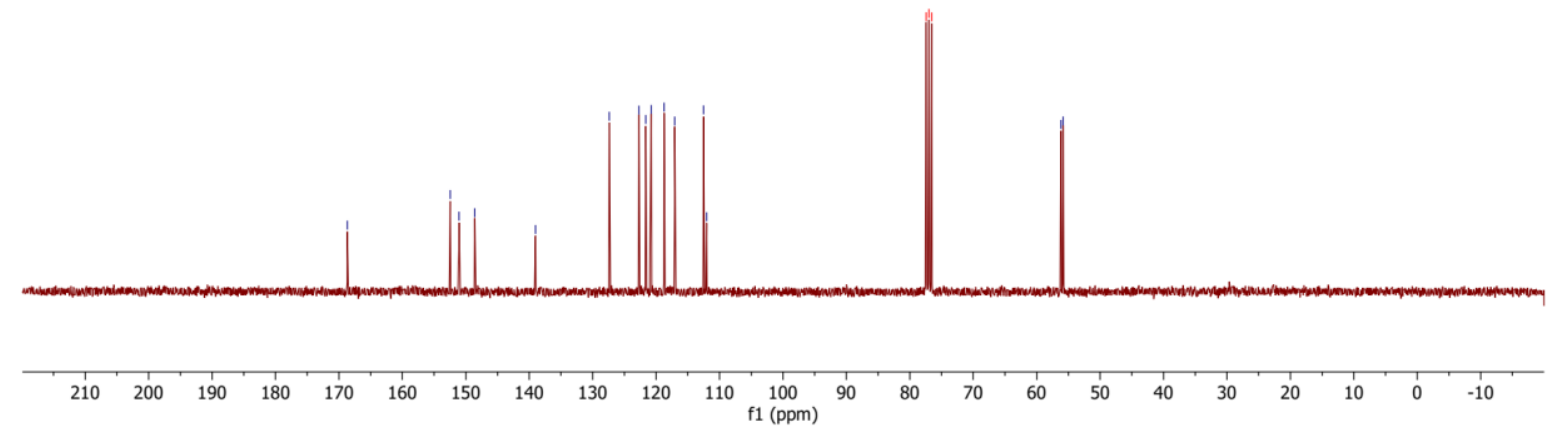



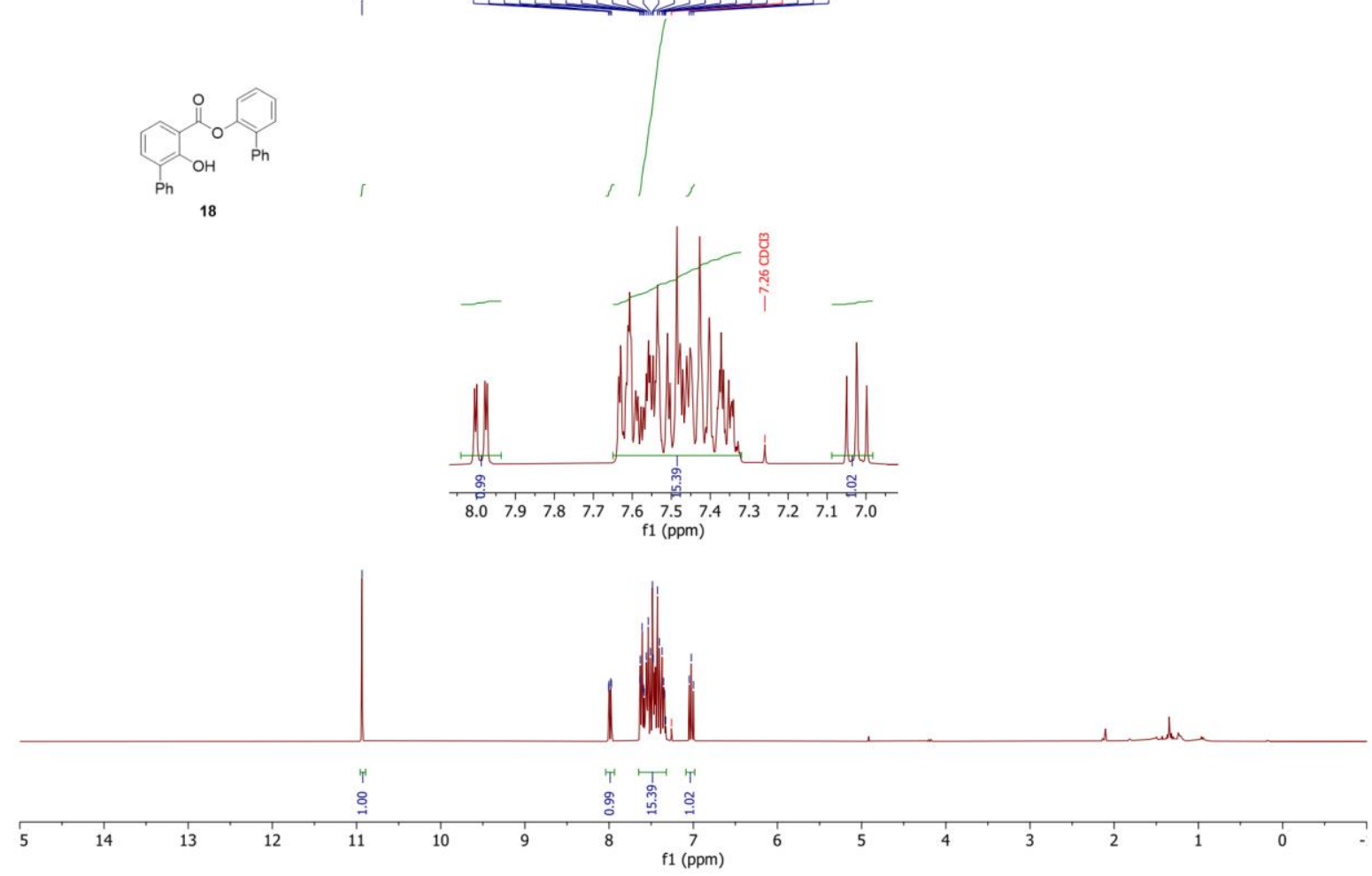

${ }^{3} \mathrm{CNMR}\left(75 \mathrm{MHz}, \mathrm{CDCl}_{3}\right)$

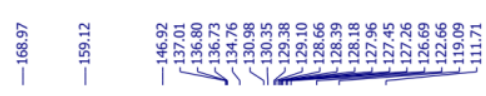<smiles>O=C(Oc1ccccc1-c1ccccc1)c1cccc(-c2ccccc2)c1O</smiles>

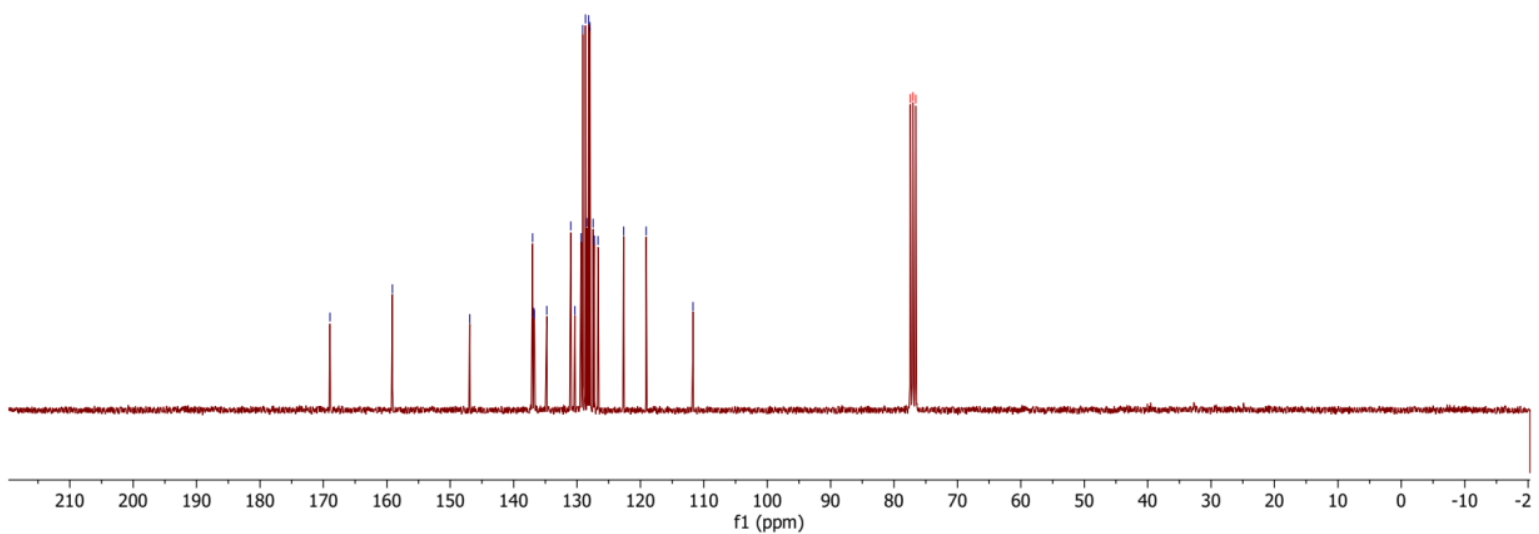

